\title{
Research Article \\ Defaultable Game Options in a Hazard Process Model
}

\section{Tomasz R. Bielecki, ${ }^{1}$ Stéphane Crépey, ${ }^{2}$ Monique Jeanblanc, ${ }^{2,3}$ and Marek Rutkowski ${ }^{4,5}$}

${ }^{1}$ Department of Applied Mathematics, Illinois Institute of Technology, Chicago, IL 60616, USA

${ }^{2}$ Département de Mathématiques, Université d'Évry Val d'Essonne, 91025 Évry Cedex, France

${ }^{3}$ Europlace Institute of Finance, Palais Brongniart-28 Place de la Bourse, 75002 Paris, France

${ }^{4}$ School of Mathematics and Statistics, University of New South Wales, Sydney, NSW 2052, Australia

${ }^{5}$ Faculty of Mathematics and Information Science, Warsaw University of Technology, 00-661 Warszawa, Poland

Correspondence should be addressed to Tomasz R. Bielecki, bielecki@iit.edu

Received 22 October 2008; Accepted 4 April 2009

Recommended by Salah-Eldin Mohammed

The valuation and hedging of defaultable game options is studied in a hazard process model of credit risk. A convenient pricing formula with respect to a reference filteration is derived. A connection of arbitrage prices with a suitable notion of hedging is obtained. The main result shows that the arbitrage prices are the minimal superhedging prices with sigma martingale cost under a risk neutral measure.

Copyright (C) 2009 Tomasz R. Bielecki et al. This is an open access article distributed under the Creative Commons Attribution License, which permits unrestricted use, distribution, and reproduction in any medium, provided the original work is properly cited.

\section{Introduction}

The goal of this work is to analyze valuation and hedging of defaultable contracts with game option features within a hazard process model of credit risk. Our motivation for considering American or game clauses together with defaultable features of an option is not that much a quest for generality, but rather the fact that the combination of early exercise features and defaultability is an intrinsic feature of some actively traded assets. It suffices to mention here the important class of convertible bonds, which were studied by, among others, Andersen and Buffum [1], Ayache et al. [2], Bielecki et al. [3, 4], Davis and Lischka [5], Kallsen and Kühn [6], and Kwok and Lau [7].

In Bielecki et al. [3], we formally defined a defaultable game option, that is, a financial contract that can be seen as an intermediate case between a general mathematical concept of a game option and much more specific convertible bond with credit risk. We concentrated 
there on developing a fairly general framework for valuing such contracts. In particular, building on results of Kifer [8] and Kallsen and Kühn [6], we showed that the study of an arbitrage price of a defaultable game option can be reduced to the study of the value process of the related Dynkin game under some risk-neutral measure $\mathbb{Q}$ for the primary market model. In this stochastic game, the issuer of a game option plays the role of the minimizer and the holder of the maximizer. In [3], we dealt with a general market model, which was assumed to be arbitrage-free, but not necessarily complete, so that the uniqueness of a riskneutral (or martingale) measure was not postulated. In addition, although the default time was introduced, it was left largely unspecified. An explicit specification of the default time will be an important component of the model considered in this work.

As is well known, there are two main approaches to modeling of default risk: the structural approach and the reduced-form approach. In the latter approach, also known as the hazard process approach, the default time is modeled as an exogenous random variable with no reference to any particular economic background. One may object to reduced-form models for their lack of clear reference to economic fundamentals, such as the firm's asset-todebt ratio. However, the possibility of choosing various parameterizations for the coefficients and calibrating these parameters to any set of CDS spreads and/or implied volatilities makes them very versatile modeling tools, well suited to price and hedge derivatives consistently with plain-vanilla instruments. It should be acknowledged that structural models, with their sound economic background, are better suited for inference of reliable debt information, such as: risk-neutral default probabilities or the present value of the firm's debt, from the equities, which are the most liquid among all financial instruments. The structure of these models, as rich as it may be (and which can include a list of factors such as stock, spreads, default status, and credit events) never rich enough to yield consistent prices for a full set of CDS spreads and/or implied volatilities of related options. As we ultimately aim to specify models for pricing and hedging contracts with optional features (such as convertible bonds), we favor the reduced-form approach in the sequel.

\subsection{Outline of the Paper}

From the mathematical perspective, the goal of the present paper is twofold. First, we wish to specialize our previous valuation results to the hazard process setup, that is, to a version of the reduced-form approach, which is slightly more general than the intensity-based setup. Hence we postulate that filtration $\mathbb{G}$ modeling the information flow for the primary market admits the representation $\mathbb{G}=\mathbb{H} \vee \mathbb{F}$, where the filtration $\mathbb{H}$ is generated by the default indicator process $H_{t}=1_{\left\{t \geq \tau_{d}\right\}}$ and $\mathbb{F}$ is some reference filtration. The main tool employed in this section is the effective reduction of the information flow from the full filtration $\mathbb{G}$ to the reference filtration $\mathbb{F}$. The main results in this part are Theorems 3.7 and 3.8, which give convenient pricing formulae with respect to the reference filtration $\mathbb{F}$.

The second goal is to study the issue of hedging of a defaultable game option in the hazard process setup. Some previous attempts to analyze hedging strategies for defaultable convertible bonds were done by Andersen and Buffum [1] and Ayache et al. [2], who worked directly with suitable variational inequalities within the Markovian intensity-based setup.

Our preliminary results for hedging strategies in a hazard process setup, Propositions 4.1 and 4.3, can be informally stated as follows: under the assumption that a related doubly reflected BSDE admits a solution $(\Theta, M, K)$ under some risk-neutral measure $\mathbb{Q}$, for which various sets of sufficient conditions are given in literature, the state-process $\Theta$ of the solution 
is the minimal (pre-default) super-hedging price up to a $(\mathbb{G}, \mathbb{Q}$ )-sigma (or local) martingale cost process. More specific properties of hedging strategies are subsequently analyzed in Propositions 4.13 and 4.15, in which we resort to suitable Galtchouk-Kunita-Watanabe decompositions of a solution to the related doubly reflected BSDE and discounted prices of primary assets with respect to various risk factors corresponding to systematic, idiosyncratic and event risks. It is noteworthy that these decompositions, though seemingly rather abstract in a general setup considered here, are by no means artificial. On the contrary, they arise naturally in the context of particular Markovian models that are studied in the followup paper by Bielecki et al. [4,9]. We conclude the paper by briefly commenting on some alternative approaches to hedging of defaultable game options.

\subsection{Conventions and Standing Notation}

Throughout this paper, we use the concept of the vector stochastic integral, denoted as $\int H d X$, as opposed to a more restricted notion of the component-wise stochastic integral, which is defined as the sum $\sum_{i=1}^{d} \int H^{i} d X^{i}$ of integrals with respect to one-dimensional integrators $X^{i}$. For a detailed exposition of the vector stochastic integration, we refer to Shiryaev and Cherny [10] (see also Chatelain and Stricker [11] and Jacod [12]). Given a stochastic basis satisfying the usual conditions, an $\mathbb{R}^{d}$-valued semimartingale integrator $X$ and an $\mathbb{R}^{1 \otimes d}$-valued (row vector) predictable integrand $H$, the notion of the vector stochastic integral $\int H d X$ allows one to take into account possible "interferences" of local martingale and finite variation components of a (scalar) integrator process, or of different components of a multidimensional integrator process. Well-defined vector stochastic integrals include, in particular, all integrals with a predictable and locally bounded integrand (e.g., any integrand of the form $H=Y_{-}$ where $Y$ is an adapted càdlàg process, see He et al. [13, Theorem 7.7]). The usual properties of stochastic integral, such as: linearity, associativity, invariance with respect to equivalent changes of measures and with respect to inclusive changes of filtrations, are known to hold for the vector stochastic integral. Moreover, unlike other kinds of stochastic integrals, vector stochastic integrals form a closed space in a suitable topology. This feature makes them well adapted to many problems arising in the mathematical finance, such as Fundamental Theorems of Asset Pricing (see, e.g., Delbaen and Schachermayer [14] or Shiryaev and Cherny [10]).

By default, we denote by $\int_{0}^{t}$ the integrals over $(0, t]$. Otherwise, we explicitly specify the domain of integration as a subscript of $\int$. Note also that, depending on the context, $\tau$ will stand either for a generic stopping time or it will be given as $\tau=\tau_{p} \wedge \tau_{c}$ for some specific stopping times $\tau_{c}$ and $\tau_{p}$. Finally, we consider the right-continuous and completed versions of all filtrations, so that they satisfy the so-called "usual conditions."

\section{Semimartingale Setup}

After recalling some fundamental valuation results from [3], we will examine basic features of hedging strategies for defaultable game options that are valid in a general semimartingale setup. The important special case of a hazard process framework is studied in the next section.

We assume throughout that the evolution of the underlying primary market is modeled in terms of stochastic processes defined on a filtered probability space $(\Omega, \mathbb{G}, \mathbb{P})$, where $\mathbb{P}$ denotes the statistical probability measure. 
Specifically, we consider a primary market composed of the savings account and of $d$ risky assets, such that, given a finite horizon date $T>0$ :

(i) the discount factor process $\beta$, that is, the inverse of the savings account, is a $\mathbb{G}$ adapted, finite variation, positive, continuous and bounded process,

(ii) the risky assets are $\mathbb{G}$-semimartingales with càdlàg sample paths.

The primary risky assets, with $\mathbb{R}^{d}$-valued price process $X$, pay dividends, whose cumulative value process, denoted by $\Phi$, is assumed to be a $\mathbb{G}$-adapted, càdlàg and $\mathbb{R}^{d}$-valued process of finite variation. Given the price process $X$, we define the cumulative price $\widehat{X}$ of primary risky assets as

$$
\widehat{X}_{t}=X_{t}+\beta_{t}^{-1} \int_{[0, t]} \beta_{u} d \Phi_{u}
$$

In the financial interpretation, the last term in (2.1) represents the current value at time $t$ of all dividend payments from the assets over the period $[0, t]$, under the assumption that all dividends are immediately reinvested in the savings account. We assume that the primary market model is free of arbitrage opportunities, though presumably incomplete. In view of the First Fundamental Theorem of Asset Pricing (cf. [10, 14]), and accounting in particular for the dividends, this means that there exists a risk-neutral measure $\mathbb{Q} \in \mathcal{M}$, where $\mathcal{M}$ denotes the set of probability measures $\mathbb{Q} \sim \mathbb{P}$ for which $\beta \widehat{X}$ is a sigma martingale with respect to $\mathbb{G}$ under $\mathbb{Q}$ (for the definition of a sigma martingale, see [10, Definition 1.9]). The following well-known properties of sigma martingales will be used in the sequel.

Proposition 2.1 (see $[10,15,16]$ ). (i) The class of sigma martingales is a vector space containing all local martingales. It is stable with respect to (vector) stochastic integration, that is, if $Y$ is a sigma martingale and $H$ is a (predictable) $Y$-integrable process then the (vector) stochastic integral $\int H d Y$ is a sigma martingale.

(ii) Any locally bounded sigma martingale is a local martingale, and any bounded from below sigma martingale is a supermartingale.

Remark 2.2. In the same vein, we recall that stochastic integration of predictable, locally bounded integrands preserves local martingales (see, e.g., Protter [16]).

We now introduce the concept of a dividend paying game option (see also Kifer [8]). In broad terms, a dividend paying game option, with the inception date 0 and the maturity date $T$, is a contract with the following cash flows that are paid by the issuer of the contract and received by its holder:

(i) a dividend stream with the cumulative dividend at time $t$ denoted by $D_{t}$,

(ii) a terminal put payment $L_{t}$ made at time $t=\tau_{p}$ if $\tau_{p} \leq \tau_{c}$ and $\tau_{p}<T$; time $\tau_{p}$ is called the put time and is chosen by the holder,

(iii) a terminal call payment $U_{t}$ made at time $t=\tau_{c}$ provided that $\tau_{c}<\tau_{p} \wedge T$; time $\tau_{c}$, known as the call time, is chosen by the issuer and may be subject to the constraint that $\tau_{c} \geq \bar{\tau}$, where $\bar{\tau}$ is the lifting time of the call protection,

(iv) a terminal payment at maturity $\xi$ made at maturity date $T$ provided that $T \leq \tau_{p} \wedge \tau_{c}$. 
The (possibly random) time $\bar{\tau}$ in (iii) is used to model the restriction that the issuer of a game option may be prevented from making a call on some random time interval $[0, \bar{\tau})$.

Of course, there is also the initial cash flow, namely, the purchasing price of the contract, which is paid at the initiation time by the holder and received by the issuer.

Let us now be given an $[0,+\infty]$-valued $\mathbb{G}$-stopping time $\tau_{d}$ representing the default time of a reference entity, with default indicator process $H_{t}=1_{\left\{\tau_{d} \leq t\right\}}$. A defaultable dividend paying game option is a dividend paying game option such that the contract is terminated at $\tau_{d}$, if it has not been put or called and has not matured before. In particular, there are no more cash flows related to this contract after the default time. In this setting, the dividend stream $D$ is assumed to include a possible recovery payment made at the default time.

We are interested in the problem of the time evolution of an arbitrage price of the game option. Therefore, we formulate the problem in a dynamic way by pricing the game option at any time $t \in[0, T]$. We write $\mathcal{G}_{T}^{t}$ to denote the set of all $\mathbb{G}$-stopping times with values in $[t, T]$ and we let $\overline{\mathcal{G}}_{T}^{t}$ stand for the set $\left\{\tau \in \mathcal{G}_{T}^{t} ; \tau \wedge \tau_{d} \geq \bar{\tau} \wedge \tau_{d}\right\}$, where the lifting time of a call protection $\bar{\tau}$ belongs to $\mathcal{G}_{T}^{0}$.

We are now in the position to state the formal definition of a defaultable game option.

Definition 2.3. A defaultable game option with lifting time of the call protection $\bar{\tau} \in \mathcal{G}_{T}^{0}$ is a game option with the ex-dividend cumulative discounted cash flows $\beta_{t} \pi\left(t ; \tau_{p}, \tau_{c}\right)$ given by the formula, for any $t \in[0, T]$ and $\left(\tau_{p}, \tau_{c}\right) \in \mathcal{G}_{T}^{t} \times \overline{\mathcal{G}}_{T}^{t}$,

$$
\beta_{t} \pi\left(t ; \tau_{p}, \tau_{c}\right)=\int_{t}^{\tau} \beta_{u} d D_{u}+1_{\left\{\tau<\tau_{d}\right\}} \beta_{\tau}\left(1_{\left\{\tau=\tau_{p}<T\right\}} L_{\tau p}+1_{\left\{\tau<\tau_{p}\right\}} U_{\tau_{c}}+1_{\{\tau=T\}} \xi\right)
$$

where $\tau=\tau_{p} \wedge \tau_{c}$ and

(i) the dividend process $D=\left(D_{t}\right)_{t \in[0, T]}$ equals

$$
D_{t}=\int_{[0, t]}\left(1-H_{u}\right) d C_{u}+\int_{[0, t]} R_{u} d H_{u}=C_{\tau-1} 1_{\{t \geq \tau\}}+C_{t} 1_{\{t<\tau\}}+R_{\tau} 1_{\{t \geq \tau\}},
$$

for some coupon process $C=\left(C_{t}\right)_{t \in[0, T]}$, which is a $\mathbb{G}$-predictable, real-valued, càdlàg process with bounded variation, and some real-valued, $\mathbb{G}$-predictable recovery process $R=\left(R_{t}\right)_{t \in[0, T]}$,

(ii) the put payment $L=\left(L_{t}\right)_{t \in[0, T]}$ and the call payment $U=\left(U_{t}\right)_{t \in[0, T]}$ are $\mathbb{G}$-adapted, real-valued, càdlàg processes,

(iii) the inequality $L_{t} \leq U_{t}$ holds for every $t \in\left[\tau_{d} \wedge \bar{\tau}, \tau_{d} \wedge T\right)$,

(iv) the payment at maturity $\xi$ is a $\mathcal{G}_{T}$-measurable, real-valued random variable.

The following result easily follows from Definition 2.3.

Lemma 2.4. (i) For any $t$ and $\left(\tau_{p}, \tau_{c}\right) \in \mathcal{G}_{T}^{t} \times \overline{\mathcal{G}}_{T}^{t}$, the random variable $\pi\left(t ; \tau_{p}, \tau_{c}\right)$ is $\mathcal{G}_{\tau \wedge \tau_{d}}{ }^{-}$ measurable.

(ii) For any $\left(\tau_{p}, \tau_{c}\right) \in \mathcal{G}_{T}^{0} \times \overline{\mathcal{G}}_{T}^{0}$, the processes $\pi\left(0 ; \cdot, \tau_{c}\right)$ and $\pi\left(0 ; \tau_{p}, \cdot\right)$ are $\mathbb{G}$-adapted. 
We further assume that $R, L$, and $\xi$ are bounded from below, so that there exists a constant $c$ such that, for every $t \in[0, T]$,

$$
\beta_{t} \widehat{\mathcal{L}}_{t}:=\int_{[0, t]} \beta_{u} d D_{u}+1_{\left\{t<\tau_{d}\right\}} \beta_{t}\left(1_{\{t<T\}} L_{t}+1_{\{t=T\}} \xi\right) \geq-c
$$

Symmetrically, we should sometimes additionally assume that $R, U$, and $\xi$ are bounded (from below and from above), or that (2.4) is supplemented by the inequality, for every $t \in[0, T]$,

$$
\beta_{t} \hat{\mathcal{U}}_{t}:=\int_{[0, t]} \beta_{u} d D_{u}+1_{\left\{t<\tau_{d}\right\}} \beta_{t}\left(1_{\{t<T\}} U_{t}+1_{\{t=T\}} \xi\right) \leq c
$$

\subsection{Valuation of a Defaultable Game Option}

We will state the following fundamental pricing result without proof, referring the interested reader to [3, Proposition 3.1 and Theorem 4.1] for more details. The goal is to characterize the set of arbitrage ex-dividend prices of a game option in terms of values of related Dynkin games (for the general theory of Dynkin games, see, e.g., Dynkin [17], Kifer [18], and Lepeltier and Maingueneau [19]). The notion of an arbitrage price of a game option referred to in Theorem 2.5 is the dynamic notion of arbitrage price for game options, as defined in Kallsen and Kühn [6], and extended in [3] to the case of dividend-paying primary assets and dividend-paying game options by resorting to the transformation of prices into cumulative prices. Note that in the sequel, the statement " $\left(\Pi_{t}\right)_{t \in[0, T]}$ is an arbitrage price for the game option" is in fact to be understood as " $\left(X_{t}, \Pi_{t}\right)_{t \in[0, T]}$ is an arbitrage price for the extended market consisting of the primary market and the game option."

Theorem 2.5 (Arbitrage price of a defaultable game option). Assume that a process $\Pi$ is a $\mathbb{G}$ semimartingale and there exists $\mathbb{Q} \in \mathcal{M}$ such that $\Pi$ is the value of the Dynkin game related to a game option, meaning that

$$
\begin{aligned}
& \underset{\tau_{p} \in \mathcal{G}_{T}^{t}}{\operatorname{ess} \sup } \operatorname{essinf} \operatorname{inc}_{\tau_{c}} \overline{\mathcal{G}}_{T}^{t} \\
& \quad=\prod_{\mathbb{Q}}\left(\pi\left(t ; \tau_{p}, \tau_{c}\right) \mid \mathcal{G}_{t}\right) \\
& \quad \Pi_{t}=\underset{\tau_{c} \in \overline{\mathcal{G}}_{T}^{t}}{\operatorname{essinf}} \operatorname{ess} \sup _{\tau_{p} \in \mathcal{G}_{T}^{t}} \mathbb{E}_{\mathbb{Q}}\left(\pi\left(t ; \tau_{p}, \tau_{c}\right) \mid \mathcal{G}_{t}\right), \quad t \in[0, T] .
\end{aligned}
$$

Then $\Pi$ is an arbitrage ex-dividend price of the game option, called the $\mathbb{Q}$-price of the game option. The converse holds true (thus any arbitrage price is a $\mathbb{Q}$-price for some $\mathbb{Q} \in \mathcal{M}$ ) under the following integrability assumption

$$
\underset{\mathbb{Q} \in \mathcal{M}}{\operatorname{essup}} \mathbb{E}_{\mathbb{Q}}\left[\sup _{t \in[0, T]}\left(\int_{[0, t]} \beta_{u} d D_{u}+1_{\left\{t<\tau_{d}\right\}} \beta_{t}\left(1_{\{t<T\}} L_{t}+1_{\{t=T\}} \xi\right)\right) \mid \mathcal{G}_{0}\right]<\infty \text {, a.s. }
$$

Note that defaultable American (or European) options can be seen as special cases of defaultable game options. 
Definition 2.6. A defaultable American option is a defaultable game option with $\bar{\tau}=T$. A defaultable European option is a defaultable American option such that the process $\beta \widehat{\perp}$ (cf. (2.4)) attains its maximum at $T$, that is, $\beta_{t} \widehat{\mathcal{L}}_{t} \leq \beta_{T} \widehat{\mathcal{L}}_{T}$ for every $t \in[0, T]$.

In view of Theorem 2.5, the cash flows $\phi(t)$ of a defaultable European option can be redefined by

$$
\beta_{t} \phi(t)=\int_{t}^{T} \beta_{u} d D_{u}+1_{\left\{\tau_{d}>T\right\}} \beta_{T} \xi, \quad t \in[0, T]
$$

\subsection{Hedging of a Defaultable Game Option}

We adopt the definition of hedging game options stemming from successive developments, starting from the hedging of American options examined by Karatzas [20], and subsequently followed by El Karoui and Quenez [21], Kifer [8], Ma and Cvitanić [22], and Hamadène [23]. One of our goals is to show that this definition is consistent with the concept of arbitrage valuation of a defaultable game option in the sense of Kallsen and Kühn [6].

Recall that $X$ (resp., $\widehat{X}$ ) is the price process (resp., cumulative price process) of primary traded assets, as given by (2.1). The following definitions are standard, accounting for the dividends on the primary market.

Definition 2.7. By a (self-financing) primary trading strategy we mean any pair $\left(V_{0}, \zeta\right)$ such that

(i) $V_{0}$ is a $\mathcal{G}_{0}$-measurable real-valued random variable representing the initial wealth,

(ii) $\zeta$ is an $\mathbb{R}^{1 \otimes d}$-valued, $\beta \hat{X}$-integrable process representing holdings in primary risky assets.

Remark 2.8. The reason why we do not assume that $\mathcal{G}_{0}$ is trivial (which would, of course, simplify several statements) is that we apply our results in the subsequent work [4] to situations, where this assumption fails to hold (e.g., when studying convertible bonds with a positive call notice period).

Definition 2.9. The wealth process $V$ of a primary trading strategy $\left(V_{0}, \zeta\right)$ is given by the formula, for $t \in[0, T]$,

$$
\beta_{t} V_{t}=\beta_{0} V_{0}+\int_{0}^{t} \zeta_{u} d\left(\beta_{u} \widehat{X}_{u}\right)
$$

Given the wealth process $V$ of a primary strategy $\left(V_{0}, \zeta\right)$, we uniquely specify a $\mathbb{G}$ optional process $\zeta^{0}$ by setting

$$
V_{t}=\zeta_{t}^{0} \beta_{t}^{-1}+\zeta_{t} X_{t}, \quad t \in[0, T]
$$

The process $\zeta^{0}$ represents the number of units held in the savings account at time $t$, when we start from the initial wealth $V_{0}$ and we use the strategy $\zeta$ in the primary risky assets. Recall that we denote $\tau=\tau_{p} \wedge \tau_{c}$. 
Definition 2.10. Consider the game option with the ex-dividend cumulative discounted cash flows $\beta \pi$ given by (2.2).

(i) An issuer hedge with cost process $\rho$ is represented by a quadruplet $\left(V_{0}, \zeta, \rho, \tau_{c}\right)$ such that

(a) $\left(V_{0}, \zeta\right)$ is a primary strategy with the wealth process $V$ given by $(2.9)$,

(b) a cost process $\rho$ is a real-valued, càdlàg $\mathbb{G}$-semimartingale with $\rho_{0}=0$,

(c) a (fixed) call time $\tau_{c}$ belongs to $\overline{\mathcal{G}}_{T}^{0}$,

(d) the following inequality is valid, for every put time $\tau_{p} \in \mathcal{G}_{T}^{0}$,

$$
\beta_{\tau} V_{\tau}+\int_{0}^{\tau} \beta_{u} d \rho_{u} \geq \beta_{0} \pi\left(0 ; \tau_{p}, \tau_{c}\right), \quad \text { a.s. }
$$

(ii) A holder hedge with cost process $\rho$ is a quadruplet $\left(V_{0}, \zeta, \rho, \tau_{p}\right)$ such that

(a) $\left(V_{0}, \zeta\right)$ is a primary strategy with the wealth process $V$ given by (2.9),

(b) a cost process $\rho$ is a real-valued, càdlàg $\mathbb{G}$-semimartingale with $\rho_{0}=0$,

(c) a (fixed) put time $\tau_{p}$ belongs to $\mathcal{G}_{T}^{0}$,

(d) the following inequality is valid, for every call time $\tau_{c} \in \overline{\mathcal{G}}_{T}^{0}$,

$$
\beta_{\tau} V_{\tau}+\int_{0}^{\tau} \beta_{u} d \rho_{u} \geq-\beta_{0} \pi\left(0 ; \tau_{p}, \tau_{c}\right), \quad \text { a.s. }
$$

Issuer or holder hedges at no cost (i.e., with $\rho=0$ ) are thus in effect issuer or holder superhedges. A more explicit form of condition (2.11) reads (for (2.12), we need to insert the minus sign in the right-hand side of (2.13))

$$
\begin{aligned}
V_{\tau}+ & \beta_{\tau}^{-1} \int_{0}^{\tau} \beta_{u} d \rho_{u} \\
& \geq \beta_{\tau}^{-1} \int_{0}^{\tau} \beta_{u} d D_{u}+1_{\left\{\tau<\tau_{d}\right\}}\left(1_{\left\{\tau=\tau_{p}<T\right\}} L_{\tau p}+1_{\left\{\tau<\tau_{p}\right\}} U_{\tau_{c}}+1_{\left\{\tau_{p}=\tau_{c}=T\right\}} \xi\right), \quad \text { a.s. }
\end{aligned}
$$

The left-hand side in (2.13) is the value at time $\tau$ of a strategy with a cost process $\rho$, when the players adopt their respective exercise policies $\tau_{p}$ and $\tau_{c}$, whereas the right-hand side represents the payoff to be done by the issuer, including past dividends and the recovery at default.

Remark 2.11. (i) The process $\rho$ is to be interpreted as the (running) financing cost, that is, the amount of cash added to (if $d \rho_{t} \geq 0$ ) or withdrawn from (if $d \rho_{t} \leq 0$ ) the hedging portfolio in order to get a perfect, but no longer self-financing, hedge. In the special case where $\rho$ is a $\mathbb{G}$-martingale under $\mathbb{Q}$ we thus recover the notion of mean self-financing hedge, in the sense of Schweizer [24].

(ii) Regarding the admissibility of hedging strategies (see, e.g., Delbaen and Schachermayer [14]), note that the left-hand side in formula (2.11) (discounted wealth process 
inclusive of financing costs) is bounded from below for any issuer hedge with a cost $\left(V_{0}, \zeta, \rho, \tau_{c}\right)$. Likewise, in the case of a bounded payoff $\pi$ (i.e., assuming (2.5)), the left-hand side in formula (2.12) is bounded from below for any holder hedge with a cost $\left(V_{0}, \zeta, \rho, \tau_{p}\right)$.

Obviously, the class of all hedges with semimartingale cost processes is too large for any practical purposes. Therefore, we will restrict our attention to hedges with a $\mathbb{G}$-sigma martingale cost $\rho$ under a particular risk-neutral measure $\mathbb{Q}$.

Assumption 2.12. In the sequel, we work under a fixed, but arbitrary, risk-neutral measure $\mathbb{Q} \in \mathcal{M}$.

All the measure-dependent notions like (local) martingale and compensator, implicitly refer to the probability measure $\mathbb{Q}$. In particular, we define $\mathcal{V}_{0}^{c}$ (resp., $\mho_{0}^{p}$ ) as the set of initial values $V_{0}$ for which there exists an issuer (resp., holder) hedge of the game option with the initial value $V_{0}$ (resp., $-V_{0}$ ) and with a $\mathbb{G}$-sigma martingale cost under $\mathbb{Q}$.

The following result gives some preliminary conclusions regarding the initial cost of a hedging strategy for the game option under the present, rather weak, assumptions. In Proposition 4.3, we will see that, under stronger assumptions, the infima are attained and thus we obtain equalities, rather than merely inequalities, in (2.14) and (2.15).

Lemma 2.13. (i) One has (by convention, ess inf $\emptyset=\infty$ )

$$
\underset{\tau_{c} \in \overline{\mathcal{G}}_{T}^{0}}{\operatorname{ess} \inf } \underset{\tau_{p} \in \mathcal{G}_{T}^{0}}{\operatorname{ess} \sup } \mathbb{E}_{\mathbb{Q}}\left(\pi\left(0 ; \tau_{p}, \tau_{c}\right) \mid \mathcal{G}_{0}\right) \leq \underset{V_{0} \in \mathcal{U}_{0}^{c}}{\operatorname{essinf}} V_{0} \text {, a.s. }
$$

(ii) If inequality (2.5) is valid then

$$
\underset{\tau_{p} \in \mathcal{G}_{T}^{0}}{\operatorname{ess} \sup } \underset{\tau_{c} \in \overline{\mathcal{G}}_{T}^{0}}{\operatorname{einf}} \mathbb{E}_{\mathbb{Q}}\left(\pi\left(0 ; \tau_{p}, \tau_{c}\right) \mid \mathcal{G}_{0}\right) \geq \underset{V_{0} \in \mathcal{V}_{0}^{p}}{-\operatorname{ess} \inf } V_{0}, \quad \text { a.s. }
$$

Proof. (i) Assume that for some stopping time $\bar{\tau}_{c} \in \overline{\mathcal{G}}_{T}^{0}$ the quadruplet $\left(V_{0}, \zeta, \rho, \bar{\tau}_{c}\right)$ is an issuer hedge with a $\mathbb{G}$-sigma martingale cost $\rho$ for the game option. It is easily seen from (2.9) and (2.11) that, for any stopping time $\tau_{p} \in \mathcal{G}_{T^{\prime}}^{0}$

$$
\begin{aligned}
\beta_{0} V_{0} & =\beta_{\tau_{p} \wedge \bar{\tau}_{c}} V_{\tau_{p} \wedge \bar{\tau}_{c}}-\int_{0}^{\tau_{p} \wedge \bar{\tau}_{c}} \zeta_{u} d\left(\beta_{u} \hat{X}_{u}\right) \\
& \geq \beta_{0} \pi\left(0 ; \tau_{p}, \bar{\tau}_{c}\right)-\int_{0}^{\tau_{p} \wedge \bar{\tau}_{c}}\left(\zeta_{u} d\left(\beta_{u} \hat{X}_{u}\right)+\beta_{u} d \rho_{u}\right) .
\end{aligned}
$$

In particular, by taking $\tau_{p}=t$, we obtain that, for any $t \in[0, T]$,

$$
\begin{aligned}
\beta_{0} V_{0} & =\beta_{t \wedge \bar{\tau}_{c}} V_{t \wedge \bar{\tau}_{c}}-\int_{0}^{t \wedge \bar{\tau}_{c}} \zeta_{u} d\left(\beta_{u} \widehat{X}_{u}\right) \\
& \geq \beta_{0} \pi\left(0 ; t, \bar{\tau}_{c}\right)-\int_{0}^{t \wedge \bar{\tau}_{c}}\left(\zeta_{u} d\left(\beta_{u} \widehat{X}_{u}\right)+\beta_{u} d \rho_{u}\right) .
\end{aligned}
$$


The stochastic integral $\int_{0}^{t} \zeta_{u} d\left(\beta_{u} \hat{X}_{u}\right)$ with respect to a $\mathbb{G}$-sigma martingale $\beta \hat{\mathrm{X}}$ is a $\mathbb{G}$-sigma martingale. Hence the stopped process $\int_{0}^{t \wedge \bar{\tau}_{c}} \zeta_{u} d\left(\beta_{u} \widehat{X}_{u}\right)$, as well as the process

$$
\int_{0}^{t \wedge \bar{\tau}_{c}}\left(\zeta_{u} d\left(\beta_{u} \widehat{X}_{u}\right)+\beta_{u} d \rho_{u}\right)
$$

are $\mathbb{G}$-sigma martingales. The latter process is bounded from below (this follows from (2.2)(2.4) and (2.17)), so that it is a bounded from below local martingale [15, page 216] and thus also a supermartingale. By taking conditional expectations in (2.16), we thus obtain for any stopping time $\tau_{p} \in \mathcal{G}_{T}^{0}$ (recall that $\bar{\tau}_{c}$ is fixed)

$$
\beta_{0} V_{0} \geq \mathbb{E}_{\mathbb{Q}}\left(\beta_{0} \pi\left(0 ; \tau_{p}, \bar{\tau}_{c}\right) \mid \mathcal{G}_{0}\right), \quad \forall \tau_{p} \in \mathcal{G}_{T}^{0}
$$

and thus, by the assumed positivity of the process $\beta$,

$$
V_{0} \geq \underset{\tau_{c} \in \overline{\mathcal{G}}_{T}^{0}}{\operatorname{ess} \inf } \underset{\tau_{p} \in \mathcal{G}_{T}^{0}}{\operatorname{essup}} \mathbb{E}_{\mathbb{Q}}\left(\pi\left(0 ; \tau_{p}, \tau_{c}\right) \mid \mathcal{G}_{0}\right) \text {, a.s. }
$$

The required inequality (2.14) is an immediate consequence of the last formula.

(ii) Let $\left(V_{0}, \zeta, \rho, \bar{\tau}_{p}\right)$ be a holder hedge with a $\mathbb{G}$-sigma martingale cost $\rho$ for the game option for some stopping time $\bar{\tau}_{p} \in \mathcal{G}_{T}^{0}$. Then (2.9) and (2.12) imply that, for any $t \in[\bar{\tau}, T]$,

$$
\begin{aligned}
\beta_{0} V_{0} & =\beta_{t \wedge \bar{\tau}_{p}} V_{t \wedge \bar{\tau}_{p}}-\int_{0}^{t \wedge \bar{\tau}_{p}} \zeta_{u} d\left(\beta_{u} \widehat{X}_{u}\right) \\
& \geq-\beta_{0} \pi\left(0 ; \bar{\tau}_{p}, t\right)-\int_{0}^{t \wedge \bar{\tau}_{p}}\left(\zeta_{u} d\left(\beta_{u} \hat{X}_{u}\right)+\beta_{u} d \rho_{u}\right) .
\end{aligned}
$$

Under condition (2.5), the stochastic integral in the last formula is bounded from below and thus we conclude, by the same arguments as in part (i) that it is a supermartingale. Consequently, for a fixed stopping time $\bar{\tau}_{p} \in \mathcal{G}_{T}^{0}$, we obtain

$$
\beta_{0} V_{0} \geq \mathbb{E}_{\mathbb{Q}}\left(-\beta_{0} \pi\left(0 ; \bar{\tau}_{p}, \tau_{c}\right) \mid \mathcal{G}_{0}\right) \text {, a.s., } \forall \tau_{c} \in \overline{\mathcal{G}}_{T}^{0} \text {, }
$$

so that

$$
V_{0} \geq-\underset{\tau_{p} \in \mathcal{G}_{T}^{0}}{\operatorname{ess} \sup } \operatorname{sinf}_{\tau_{c} \in \overline{\mathcal{G}}_{T}^{0}} \mathbb{E}_{\mathbb{Q}}\left(\pi\left(0 ; \tau_{p}, \tau_{c}\right) \mid \mathcal{G}_{0}\right), \quad \text { a.s., }
$$

and this in turn implies (2.15).

\section{Valuation in a Hazard Process Setup}

In order to get more explicit pricing and hedging results for defaultable game options, we will now study the so-called hazard process setup. 


\subsection{Standing Assumptions}

Given an $[0,+\infty]$-valued $\mathbb{G}$-stopping time $\tau_{d}$, we assume that $\mathbb{G}=\mathbb{H} \vee \mathbb{F}$, where the filtration $\mathbb{H}$ is generated by the process $H_{t}=1_{\left\{\tau_{d} \leq t\right\}}$ and $\mathbb{F}$ is some reference filtration. As expected, our approach will consist in effectively reducing the information flow from the full filtration $\mathbb{G}$ to the reference filtration $\mathbb{F}$.

Let $G$ stand for the process $G_{t}=\mathbb{Q}\left(\tau_{d}>t \mid \mathcal{F}_{t}\right)$ for $t \in \mathbb{R}_{+}$. The process $G$ is a (bounded) $\mathbb{F}$-supermartingale, as the optional projection on the filtration $\mathbb{F}$ of the nonincreasing process $1-H$ (see Jeulin [25]).

In the sequel, we will work under the following standing assumption.

Assumption 3.1. We assume that the process $G$ is (strictly) positive and continuous with finite variation, so that the $\mathbb{F}$-hazard process $\Gamma_{t}=-\ln \left(G_{t}\right), t \in \mathbb{R}_{+}$, is well defined and continuous with finite variation.

Remark 3.2. (i) The assumption that $G$ is continuous implies that $\tau_{d}$ is a totally inaccessible $\mathbb{G}$ stopping time (see, e.g., [26]). Moreover, $\tau_{d}$ avoids $\mathbb{F}$-stopping times, in the sense that $\mathbb{Q}\left(\tau_{d}=\right.$ $\tau)=0$ for any $\mathbb{F}$-stopping time $\tau$ (see Coculescu and Nikeghbali [27]).

(ii) If $G$ is continuous, the additional assumption that $G$ has a finite variation implies in fact that $G$ is nonincreasing (see Lemma A.1(i)). This lies somewhere between assuming further the (stronger) ( $\mathscr{l})$ Hypothesis and assuming further that $\tau_{d}$ is an $\mathbb{F}$-pseudo-stopping time (see Nikeghbali and Yor [28]). Recall that the ( $\mathscr{\ell}$ ) Hypothesis means that all local $\mathbb{F}$ martingales are local $\mathbb{G}$-martingales (see, e.g., [29]), whereas $\tau_{d}$ is an $\mathbb{F}$-pseudo-stopping time whenever all $\mathbb{F}$-local martingales stopped at $\tau_{d}$ are $\mathbb{G}$-local martingales (see Nikeghbali and Yor [28] and the appendix).

Some consequences of Assumption 3.1 useful for this work are summarized in Lemma A.1. The next definition refers to some auxiliary results, which are relegated to the appendix.

Definition 3.3. The $\mathbb{F}$-stopping time $\tilde{\tau}$, the $\mathcal{F}_{t}$-measurable random variable $\tilde{x}$ and the $\mathbb{F}$ adapted or $\mathbb{F}$-predictable process $\tilde{Y}$ introduced in Lemmas A.2 and A.4 are called the $\mathbb{F}$ representatives of $\tau, X$ and $Y$, respectively. In the context of credit risk, where $\tau_{d}$ represents the default time of a reference entity, they are also known as the pre-default values of $\tau, \chi$ and Y.

To simplify the presentation, we find it convenient to make additional assumptions. Strictly speaking, these assumptions are superfluous, in the sense that all the results below are true without Assumption 3.4. Indeed, by making use of Lemmas A.2 and A.4 and Definition 3.3, it is always possible to reduce the original problem to the case described in Assumption 3.4. Since this would make the notation heavier, without adding much value, we prefer to work under this standing assumption.

Assumption 3.4. (i) The discount factor process $\beta$ is $\mathbb{F}$-adapted.

(ii) The coupon process $C$ is $\mathbb{F}$-predictable.

(iii) The recovery process $R$ is $\mathbb{F}$-predictable.

(iv) The payoff processes $L, U$ are $\mathbb{F}$-adapted and the random variable $\xi$ is $\boldsymbol{F}_{T^{-}}$ measurable.

(v) The call protection $\bar{\tau}$ is an $\mathbb{F}$-stopping time. 


\subsection{Reduction of a Filtration}

The next lemma shows that the computation of the lower and upper value of the Dynkin games (2.6) with respect to $\mathbb{G}$-stopping times can be reduced to the computation of the lower and upper value with respect to $\mathbb{F}$-stopping times.

Lemma 3.5. One has that

$$
\begin{aligned}
& \underset{\tau_{p} \in \mathcal{G}_{T}^{t}}{\operatorname{ess} \sup } \operatorname{ess} \inf _{\tau_{c} \in \overline{\mathcal{G}}_{T}^{t}} \mathbb{E}_{\mathbb{Q}}\left(\pi\left(t ; \tau_{p}, \tau_{c}\right) \mid \mathcal{G}_{t}\right)=\underset{\tau_{p} \in \mathcal{F}_{T}^{t}}{\operatorname{ess} \sup } \operatorname{ess}_{\tau_{c} \in \overline{\mathcal{T}}_{T}^{t}} \mathbb{E}_{\mathbb{Q}}\left(\pi\left(t ; \tau_{p}, \tau_{c}\right) \mid \mathcal{G}_{t}\right),
\end{aligned}
$$

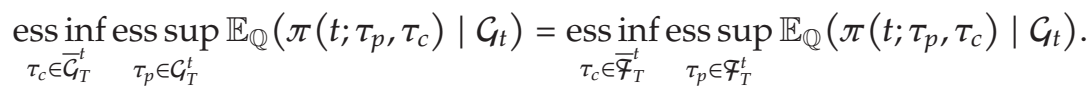

Proof. For $\left(\tau_{p}, \tau_{c}\right) \in \mathcal{G}_{T}^{t} \times \overline{\mathcal{G}}_{T}^{t}$, one has that

$$
\pi\left(t ; \tau_{p}, \tau_{c}\right)=\pi\left(t ; \tau_{p} \wedge \tau_{d}, \tau_{c} \wedge \tau_{d}\right)=\pi\left(t ; \tilde{\tau}_{p} \wedge \tau_{d}, \tilde{\tau}_{c} \wedge \tau_{d}\right)=\pi\left(t ; \tilde{\tau}_{p}, \tilde{\tau}_{c}\right)
$$

for some stopping times $\left(\widetilde{\tau}_{p}, \tilde{\tau}_{c}\right) \in \mathcal{F}_{T}^{t} \times \overline{\mathcal{F}}_{T}^{t}$, where the middle equality follows from Lemma A.4, and the other two from the definition of $\pi$. Since, clearly, $\mathcal{F}_{T}^{t} \subseteq \mathcal{G}_{T}^{t}$ and $\overline{\mathscr{F}}_{T}^{t} \subseteq \overline{\mathcal{G}}_{T}^{t}$, we conclude that the lemma is valid.

Under our assumptions, the computation of conditional expectations of cash flows $\pi\left(t ; \tau_{p}, \tau_{c}\right)$ with respect to $\mathcal{G}_{t}$ can be reduced to the computation of conditional expectations of $\mathbb{F}$-equivalent cash flows $\tilde{\pi}\left(t ; \tau_{p}, \tau_{c}\right)$ with respect to $\mathcal{F}_{t}$. Let $\alpha_{t}:=\beta_{t} \exp \left(-\Gamma_{t}\right)$ stand for the credit-risk adjusted discount factor. Note that, similarly to $\beta$, the process $\alpha$ is bounded.

Lemma 3.6. For any stopping times $\tau_{p} \in \mathcal{F}_{T}^{t}$ and $\tau_{c} \in \overline{\mathscr{F}}_{T}^{t}$ one has that

$$
\mathbb{E}_{\mathbb{Q}}\left(\pi\left(t ; \tau_{p}, \tau_{c}\right) \mid \mathcal{G}_{t}\right)=1_{\left\{t<\tau_{d}\right\}} \mathbb{E}_{\mathbb{Q}}\left(\tilde{\pi}\left(t ; \tau_{p}, \tau_{c}\right) \mid \mathcal{F}_{t}\right),
$$

where $\tilde{\pi}\left(t ; \tau_{p}, \tau_{c}\right)$ is given by, with $\tau=\tau_{p} \wedge \tau_{c}$,

$$
\alpha_{t} \tilde{\pi}\left(t ; \tau_{p}, \tau_{c}\right)=\int_{t}^{\tau} \alpha_{u}\left(d C_{u}+R_{u} d \Gamma_{u}\right)+\alpha_{\tau}\left(1_{\left\{\tau=\tau_{p}<T\right\}} L_{\tau p}+1_{\left\{\tau<\tau_{p}\right\}} U_{\tau_{c}}+1_{\{\tau=T\}} \xi\right) .
$$

Proof. Formula (3.3) is an immediate consequence of formula (2.2) and Lemma A.5.

Note that $\tilde{\pi}\left(t ; \tau_{p}, \tau_{c}\right)$ is an $\mathcal{F}_{\tau}$-measurable random variable. A comparison of formulae (2.2) and (3.4) shows that we have effectively moved our considerations from the original market subject to the default risk, in which cash flows are discounted according to the discount factor $\beta$, to the fictitious default-free market, in which cash flows are discounted according to the credit risk adjusted discount factor $\alpha$. Recall that the original cash flows $\pi\left(t ; \tau_{p}, \tau_{c}\right)$ are given as $\mathcal{G}_{\tau \wedge \tau_{d}}$-measurable random variables, whereas the $\mathbb{F}$-equivalent cash flows $\tilde{\pi}\left(t ; \tau_{p}, \tau_{c}\right)$ are manifestly $\mathcal{F}_{\tau}$-measurable and they depend on the default time $\tau_{d}$ only via the hazard process $\Gamma$. For the purpose of computation of the ex-dividend price of 
a defaultable game option these two market models are in fact equivalent. This follows from the next result, which is obtained by combining Theorem 2.5 with Lemmas 3.5 and 3.6.

Theorem 3.7 (Pre-default price of a defaultable game option). Assuming condition (2.7), let $\Pi$ be the arbitrage ex-dividend $\mathbb{Q}$-price for a game option. Then one has, for any $t \in[0, T]$,

$$
\Pi_{t}=1_{\left\{t<\tau_{d}\right\}} \tilde{\Pi}_{t}
$$

where $\tilde{\Pi}_{t}$ satisfies

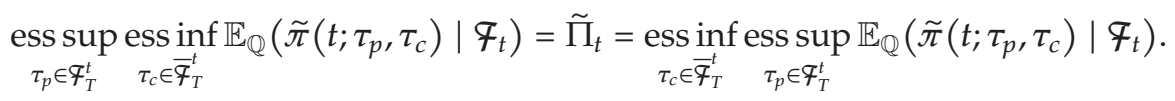

Hence the Dynkin game with cost criterion $\mathbb{E}_{\mathbb{Q}}\left(\tilde{\pi}\left(t ; \tau_{p}, \tau_{c}\right) \mid \mathcal{F}_{t}\right)$ on $\mathcal{F}_{T}^{t} \times \overline{\boldsymbol{F}}_{T}^{t}$ admits the value $\tilde{\Pi}_{t}$, which coincides with the pre-default ex-dividend price at time $t$ of the game option under the riskneutral measure $\mathbb{Q}$.

The following result is the converse of Theorem 3.7. It is an immediate consequence of Lemmas 3.5 and 3.6 and the "if" part of Theorem 2.5 (noting also that $\Pi$ defined by (3.5) is obviously a $\mathbb{G}$-semimartingale if $\widetilde{\Pi}$ is a $\mathbb{G}$-semimartingale).

Theorem 3.8. Let $\widetilde{\Pi}_{t}$ be the value of the Dynkin game with the cost criterion $\mathbb{E}_{\mathbb{Q}}\left(\tilde{\pi}\left(t ; \tau_{p}, \tau_{c}\right) \mid \mathcal{F}_{t}\right)$ on $\mathcal{F}_{T}^{t} \times \overline{\boldsymbol{F}}_{T}^{t}$, for any $t \in[0, T]$. Then $\Pi_{t}$ defined by (3.5) is the value of the Dynkin game with the cost criterion $\mathbb{E}_{\mathbb{Q}}\left(\pi\left(t ; \tau_{p}, \tau_{c}\right) \mid \mathcal{G}_{t}\right)$ on $\mathcal{G}_{T}^{t} \times \overline{\mathcal{G}}_{T}^{t}$, for any $t \in[0, T]$. If, in addition, $\widetilde{\Pi}$ is a $\mathbb{G}$-semimartingale then $\Pi$ is the arbitrage ex-dividend $\mathbb{Q}$-price for the game option.

Theorems 3.7 and 3.8 thus allow us to reduce the study of a game option to the study of Dynkin games (3.6) with respect to the reference filtration $\mathbb{F}$.

\subsection{Valuation via Doubly Reflected BSDEs}

In this section, we will characterize the arbitrage ex-dividend $\mathbb{Q}$-price of a game option as a solution to an associated doubly reflected BSDE. To this end, we first recall some auxiliary results concerning the relationship between Dynkin games and doubly reflected BSDEs.

Given an additional $\mathbb{F}$-adapted process $F$ of finite variation, we consider the following doubly reflected $B S D E$ with the data $\alpha, F, \xi, L, U, \bar{\tau}$ (see Cvitanić and Karatzas [30], Hamadène and Hassani [31], Crépey [32], Crépey and Matoussi [33], Bielecki et al. [4, 9]):

$$
\begin{gathered}
\alpha_{t} \Theta_{t}=\alpha_{T} \xi+\alpha_{T} F_{T}-\alpha_{t} F_{t}+\int_{t}^{T} \alpha_{u} d K_{u}-\int_{t}^{T} \alpha_{u} d M_{u}, \quad t \in[0, T], \\
L_{t} \leq \Theta_{t} \leq \bar{U}_{t}, \quad t \in[0, T], \\
\int_{0}^{T}\left(\Theta_{u}-L_{u}\right) d K_{u}^{+}=\int_{0}^{T}\left(\bar{U}_{u}-\Theta_{u}\right) d K_{u}^{-}=0,
\end{gathered}
$$


where the process $\bar{U}=\left(\bar{U}_{t}\right)_{t \in[0, T]}$ equals, for $t \in[0, T]$,

$$
\bar{U}_{t}=1_{\{t<\bar{\tau}\}} \infty+1_{\{t \geq \bar{\tau}\}} U_{t} .
$$

Definition 3.9. By a $(\mathbb{Q}$-)solution to the doubly reflected BSDE (3.7), we mean a triplet $(\Theta, M, K)$ such that

(i) the state process $\Theta$ is a real-valued, $\mathbb{F}$-adapted, càdlàg process,

(ii) $\int_{0} \alpha d M$ is a real-valued $\mathbb{F}$-martingale vanishing at time 0 ,

(iii) $K$ is an $\mathbb{F}$-adapted, continuous, finite variation process vanishing at time 0 ,

(iv) all conditions in (3.7) are satisfied, where in the third line $K^{+}$and $K^{-}$denote the Jordan components of $K$, and where the convention that $0 \times \pm \infty=0$ is made in the third line.

By the Jordan decomposition, we mean the decomposition $K=K^{+}-K^{-}$, where the nondecreasing continuous processes $K^{+}$and $K^{-}$vanish at time 0 and define mutually singular measures.

The state process $\Theta$ in a solution to (3.7) is clearly an $\mathbb{F}$-semimartingale. So there are obvious (though rather artificial) cases in which (3.7) does not admit a solution: it suffices to take $\bar{\tau}=0$ and $L=U$, assumed not to be an $\mathbb{F}$-semimartingale. It is also clear that a solution would not necessarily be unique if we did not impose the condition of a mutual singularity of the nonnegative measures defined by $K^{+}$and $K^{-}$(see, e.g., [31, Remark 4.1]).

Remark 3.10. In applications (see $[4,9,32,33]$ ), the input process $F$ is typically given in the form of the Lebesgue integral $\alpha F=\int \alpha f d u$ and the component $M$ of a solution to (3.7) is usually searched for in the form $M=\int Z d N+n$ for some $\mathbb{R}^{q}$-valued and real-valued squareintegrable $\mathbb{F}$-martingales $N$ and $n$ (see also Assumption 4.7 in Section 4.3). For more explicit (in particular, Markovian) specifications of the present setup and sufficient conditions for the existence and uniqueness of a solution to (3.7), the interested reader is referred to, for example, [4, 30-33].

Basically, in any model endowed with the martingale representation property, the existence (and uniqueness) of a solution to (3.7) (supplemented by suitable integrability conditions on the data and the solution) is equivalent to the so-called Mokobodski condition, namely, the existence of a quasimartingale $Z$ such that $L \leq Z \leq U$ on $[0, T]$ (see, in particular, Crépey and Matoussi [33], Hamadène and Hassani [31, Theorem 4.1], and previous works in this direction, starting with Cvitanić and Karatzas [30]). It is thus satisfied when one of the barriers is a quasimartingale and, in particular, when one of the barriers is given as $S \vee \ell$, where $S$ is an Itô-Lévy process $S$ with square-integrable special semimartingale decomposition components (see [33]) and $\ell$ is a constant in $\mathbb{R} \cup\{-\infty\}$. This framework covers, for instance, the payoff at call of a convertible bond examined in $[3,4]$.

Remark 3.11. (i) Since $K$, and thus $K^{+}$and $K^{-}$, are continuous, the minimality conditions (third line) in (3.7) are equivalent to

$$
\int_{0}^{T}\left(\Theta_{u_{-}}-L_{u-}\right) d K_{u}^{+}=\int_{0}^{T}\left(\bar{U}_{u_{-}}-\Theta_{u^{-}}\right) d K_{u}^{-}=0
$$


Indeed the related integrands here and in the third line of (3.7) differ on an at most countable set whereas the integrators define atomless measures on $[0, T]$; see, for example, [33]. In the preprint version [34] of this work, we defined more general notions of $\varepsilon$-hedges that were pertaining in the case where there may be jumps in the process $K$. Since in all existing works on doubly reflected BSDEs the process $K$ is actually found to be a continuous process (see $[4,30,31,33]$ ), we decided to impose here the continuity of $K$ in Definition 3.9 and we only consider hedges, as opposed to $\varepsilon$-hedges. Note, however, that essentially all the results of this paper can be extended to possible jumps in $K$, using the generalized notion of $\varepsilon$-hedge defined in [34], and with the minimality conditions stated as (3.9) instead of the third line in condition (3.7) of Definition 3.9.

(ii) Since $F$ is a given process, the BSDE (3.7) can be rewritten as

$$
\begin{gathered}
\alpha_{t} \widehat{\Theta}_{t}=\alpha_{T} \widehat{\xi}+\int_{t}^{T} \alpha_{u} d K_{u}-\int_{t}^{T} \alpha_{u} d M_{u}, \quad t \in[0, T], \\
\widehat{L}_{t} \leq \widehat{\Theta}_{t} \leq \widehat{U}_{t}, \quad t \in[0, T], \\
\int_{0}^{T}\left(\widehat{\Theta}_{u}-\widehat{L}_{u}\right) d K_{u}^{+}=\int_{0}^{T}\left(\widehat{U}_{u}-\widehat{\Theta}_{u}\right) d K_{u}^{-}=0,
\end{gathered}
$$

where $\widehat{\Theta}_{t}=\Theta_{t}+F_{t}, \widehat{\xi}=\xi+F_{T}, \widehat{L}_{t}=L_{t}+F_{t}$, and $\widehat{U}_{t}=\bar{U}_{t}+F_{t}$. This shows that the problem of solving (3.7) can be formally reduced to the case of $F=0$ with suitably modified reflecting barriers $\widehat{L}, \widehat{U}$ and terminal condition $\widehat{\xi}$. However, the freedom to choose the driver of a related BSDE associated with a game option is important from the point of view of applications (this is apparent in the followup papers [4, 9]; see also [34]).

(iii) In the special case where all $\mathbb{F}$-martingales are continuous and where the $\mathbb{F}$ semimartingale $F$ and the barriers $L$ and $U$ are continuous (see $[4,30,35]$ ), it is natural to look for a continuous solution of (3.7), that is, a solution of (3.7) given by a triplet of continuous processes $(\Theta, M, K)$.

(iv) In the context of a Markovian setup, the probabilistic BSDE approach may be complemented by a related analytic variational inequality approach; this issue is dealt with in the followup papers [4,9]. Note, however, that the variational inequality approach strongly relies on the BSDE approach. Moreover, a simulation method based on the BSDE is the only efficient way of numerically solving the pricing problem whenever the problem dimension (number of model factors) is greater than three or four. Indeed, in that case the computational cost of deterministic numerical schemes based on the variational inequality approach becomes prohibitive.

In order to establish a relationship between a solution to the related doubly reflected BSDE and the arbitrage ex-dividend $\mathbb{Q}$-price of the defaultable game option, we first recall the general relationship between doubly reflected BSDEs and Dynkin games with purely terminal cost, before applying this result to dividend-paying game options in the fictitious default-free market in Proposition 3.12.

Observe that if $(\Theta, M, K)$ solves (3.7) then one has, for any stopping time $\tau \in \mathcal{F}_{T}^{t}$,

$$
\alpha_{t} \Theta_{t}=\alpha_{\tau} \Theta_{\tau}+\alpha_{\tau} F_{\tau}-\alpha_{t} F_{t}+\int_{t}^{\tau} \alpha_{u} d K_{u}-\int_{t}^{\tau} \alpha_{u} d M_{u}
$$


Proposition 3.12 (Verification principle for a Dynkin game). Let $(\Theta, M, K)$ be a solution to (3.7) with $F=0$. Then $\Theta_{t}$ is the value of the Dynkin game with cost criterion $\mathbb{E}_{\mathbb{Q}}\left(\theta\left(t ; \tau_{p}, \tau_{c}\right) \mid \mathcal{F}_{t}\right)$ on $\mathcal{F}_{T}^{t} \times \overline{\mathcal{F}}_{T}^{t}$, where $\theta\left(t ; \tau_{p}, \tau_{c}\right)$ is the $\mathcal{F}_{\tau}$-measurable random variable defined by

$$
\alpha_{t} \theta\left(t ; \tau_{p}, \tau_{c}\right)=\alpha_{\tau}\left(1_{\left\{\tau=\tau_{p}<T\right\}} L_{\tau_{p}}+1_{\left\{\tau=\tau_{c}<\tau_{p}\right\}} U_{\tau_{c}}+1_{\{\tau=T\}} \xi\right),
$$

where $\tau=\tau_{p} \wedge \tau_{c}$. Moreover, for any $t \in[0, T]$, the pair of stopping times $\left(\tau_{p}^{*}, \tau_{c}^{*}\right) \in \mathcal{F}_{T}^{t} \times \overline{\boldsymbol{F}}_{T}^{t}$ given by

$$
\tau_{p}^{*}=\inf \left\{u \in[t, T] ; \Theta_{u} \leq L_{u}\right\} \wedge T, \quad \tau_{c}^{*}=\inf \left\{u \in[\bar{\tau} \vee t, T] ; \Theta_{u} \geq U_{u}\right\} \wedge T
$$

is a saddle-point of this Dynkin game, in the sense that one has, for any $\left(\tau_{p}, \tau_{c}\right) \in \mathcal{F}_{T}^{t} \times \overline{\boldsymbol{F}}_{T}^{t}$,

$$
\mathbb{E}_{\mathbb{Q}}\left(\theta\left(t ; \tau_{p}, \tau_{c}^{*}\right) \mid \mathscr{F}_{t}\right) \leq \Theta_{t} \leq \mathbb{E}_{\mathbb{Q}}\left(\theta\left(t ; \tau_{p}^{*}, \tau_{c}\right) \mid \mathscr{F}_{t}\right)
$$

Proof. Except for the presence of $\bar{\tau}$, the result is standard (see, e.g., Lepeltier and Maingueneau [19]). Let us first check that the right-hand side inequality in (3.14) is valid for any $\tau_{c} \in \overline{\bar{F}}_{T}^{t}$. Let $\tau^{*}$ denote $\tau_{p}^{*} \wedge \tau_{c}$. By the definition of $\tau_{p}^{*}$ and continuity of $K^{+}$, we see that $K^{+}$equals 0 on $\left[t, \tau^{*}\right]$. Since $K^{-}$is nondecreasing, (3.11) is applied to yield

$$
\alpha_{t} \Theta_{t} \leq \alpha_{\tau^{*}} \Theta_{\tau^{*}}-\int_{t}^{\tau^{*}} \alpha_{u} d M_{u}
$$

Taking conditional expectations (recall that $\int_{t} \alpha_{u} d M_{u}$ is an $\mathbb{F}$-martingale), and using also the facts that $\Theta_{\tau_{p}^{*}} \leq L_{\tau_{p}^{*}}$ if $\tau_{p}^{*}<T, \Theta_{\tau_{p}^{*}}=\xi$ if $\tau_{p}^{*}=T$ and $\Theta_{\tau_{\mathcal{C}}} \leq U_{\tau_{\mathcal{C}}}$ (recall that $\tau_{c} \in \overline{\boldsymbol{F}}_{T}^{t}$, so that $\tau_{\mathcal{c}} \geq \bar{\tau}$ and $\left.\bar{U}_{\tau_{c}}=U_{\tau_{c}}\right)$, we obtain

$$
\begin{aligned}
\alpha_{t} \Theta_{t} & \leq \mathbb{E}_{\mathbb{Q}}\left(\alpha_{\tau^{*}} \Theta_{\tau^{*}} \mid \mathcal{F}_{t}\right) \\
& \leq \mathbb{E}_{\mathbb{Q}}\left(\alpha_{\tau^{*}}\left(1_{\left\{\tau^{*}=\tau_{p}^{*}<T\right\}} L_{\tau_{p}^{*}}+1_{\left\{\tau^{*}=\tau_{c}<\tau_{p}^{*}\right\}} U_{\tau_{c}}+1_{\left\{\tau^{*}=T\right\}} \xi\right) \mid \mathcal{F}_{t}\right) .
\end{aligned}
$$

We conclude that $\Theta_{t} \leq \mathbb{E}_{\mathbb{Q}}\left(\theta\left(t ; \tau_{p}^{*}, \tau_{c}\right) \mid \mathcal{F}_{t}\right)$ for any $\tau_{c} \in \overline{\mathscr{F}}_{T}^{t}$. This completes the proof of the right-hand side inequality in (3.14). The left-hand side inequality can be shown similarly. It is in fact standard, since it does not involve $\bar{\tau}$, and thus the details are left to the reader.

Let us now apply Proposition 3.12 to a defaultable game option. To this end, we first rewrite (3.4) as follows

$$
\alpha_{t} \tilde{\pi}\left(t ; \tau_{p}, \tau_{c}\right)=\alpha_{\tau} \bar{F}_{\tau}-\alpha_{t} \bar{F}_{t}+\alpha_{\tau}\left(1_{\left\{\tau=\tau_{p}<T\right\}} L_{\tau p}+1_{\left\{\tau<\tau_{p}\right\}} U_{\tau_{c}}+1_{\{\tau=T\}} \xi\right),
$$


where

$$
\bar{F}_{t}:=\alpha_{t}^{-1} \int_{[0, t]} \alpha_{u} d \bar{D}_{u} \quad \text { with } \bar{D}_{t}:=\int_{[0, t]} d C_{u}+R_{u} d \Gamma_{u}
$$

Let us denote by $(\overline{\mathcal{E}})$ equation (3.10) with $F_{t}=\bar{F}_{t}$, that is,

$$
\begin{gathered}
\alpha_{t} \bar{\Theta}_{t}=\alpha_{T} \widehat{\xi}+\int_{t}^{T} \alpha_{u} d K_{u}-\int_{t}^{T} \alpha_{u} d M_{u}, \quad t \in[0, T], \\
\widehat{L}_{t} \leq \bar{\Theta}_{t} \leq \widehat{U}_{t}, \quad t \in[0, T], \\
\int_{0}^{T}\left(\bar{\Theta}_{u}-\widehat{L}_{u}\right) d K_{u}^{+}=\int_{0}^{T}\left(\widehat{U}_{u}-\bar{\Theta}_{u}\right) d K_{u}^{-}=0,
\end{gathered}
$$

with $\widehat{\xi}=\xi+\bar{F}_{T}, \widehat{L}_{t}=L_{t}+\bar{F}_{t}$, and $\widehat{U}_{t}=\bar{U}_{t}+\bar{F}_{t}$.

Assumption 3.13. The doubly reflected BSDE $(\overline{\mathcal{E}})$ admits a solution $(\bar{\Theta}, M, K)$.

Let us stress that Assumption 3.13, heroic as it may seem in the general hazard process setup, is in fact a plausible assumption in any reasonable application one may think of (cf. the comments following Definition 3.9).

We denote, for $t \in[0, T]$,

$$
\begin{gathered}
\tilde{\Pi}_{t}=\bar{\Theta}_{t}-\bar{F}_{t}, \quad \Pi_{t}=1_{\left\{t<\tau_{d}\right\}} \tilde{\Pi}_{t}, \quad \widehat{\Pi}_{t}=\Pi_{t}+\beta_{t}^{-1} \int_{[0, t]} \beta_{u} d D_{u} \\
m_{t}=\beta_{t} \widehat{\Pi}_{t}+\int_{0}^{t \wedge \tau_{d}} \beta_{u} d K_{u} .
\end{gathered}
$$

The following lemma is crucial in what follows (Lemma 3.14(i) is actually the key of the proof of Proposition 4.1 below).

Lemma 3.14. (i) The process $m$ given by $(3.20)$ is $\mathbb{G}$-martingale stopped at $\tau_{d}$.

(ii) The process $\Pi$ is a $\mathbb{G}$-semimartingale.

(iii) The process $\beta \widehat{\Pi}$ is a special $\mathbb{G}$-semimartingale.

Proof. (i) The triplet $(\tilde{\Pi}, M, K)$ satisfies (3.7) with $F$ given by $\bar{F}$ in (3.18). Therefore, for every $t \in[0, T]$,

$$
\alpha_{t} \tilde{\Pi}_{t}=\alpha_{T} \xi+\int_{t}^{T} \alpha_{u} d \bar{D}_{u}+\int_{t}^{T} \alpha_{u} d K_{u}-\int_{t}^{T} \alpha_{u} d M_{u}
$$

and thus

$$
\int_{0}^{t} \alpha_{u} d M_{u}=\alpha_{t} \tilde{\Pi}_{t}-\alpha_{0} \tilde{\Pi}_{0}+\int_{0}^{t} \alpha_{u} d K_{u}+\int_{0}^{t} \alpha_{u} d \bar{D}_{u}
$$


Using Lemma A.5, it is easy to check that one has, for any $0 \leq t \leq u \leq T$,

$$
1_{\left\{t<\tau_{d}\right\}} e^{\Gamma_{t}} \mathbb{E}_{\mathbb{Q}}\left(\int_{t}^{u} \alpha_{v} d M_{v} \mid \mathcal{F}_{t}\right)=\mathbb{E}_{\mathbb{Q}}\left(m_{u}-m_{t} \mid \mathcal{G}_{t}\right)
$$

Since the integral $\int_{t} \alpha_{v} d M_{v}$ is an $\mathbb{F}$-martingale, the process $m$ is a $\mathbb{G}$-martingale. It is also clear that it is stopped at $\tau_{d}$.

(ii) In view of (3.19), (3.20) and part (i), the process $\Pi$ is clearly a $\mathbb{G}$-semimartingale.

(iii) By (3.20), one has that

$$
\beta_{t} \widehat{\Pi}_{t}=m_{t}-\int_{0}^{t \wedge \tau_{d}} \beta_{u} d K_{u}
$$

where $m$ is a $\mathbb{G}$-martingale, by (i), and where the second term in the right-hand side is a $\mathbb{G}$-adapted and continuous (hence $\mathbb{G}$-predictable) processes of finite variation.

Remark 3.15. In view of (3.24) and since $K$ is continuous, the process $m$ given by (3.20) can equivalently be redefined as the canonical $\mathbb{G}$-local martingale component of the discounted cumulative $\mathbb{Q}$-value process $\beta \widehat{\Pi}$. The processes $m$ and $\beta \widehat{\Pi}$ are easily seen to coincide on the random interval $\left[0, \tau_{c}^{*} \wedge \tau_{p}^{*} \wedge \tau_{d} \wedge T\right]$. Therefore, both $m$ and $\beta \widehat{\Pi}$ can be interpreted on this interval as the discounted cumulative $\mathbb{Q}$-value of a defaultable game option.

The following result establishes a useful connection between $(\bar{\Theta}, M, K)$ and the arbitrage ex-dividend $\mathbb{Q}$-price of the defaultable game option.

Proposition 3.16 (Verification principle for a defaultable game option). The process $\Pi$ is the arbitrage ex-dividend $\mathbb{Q}$-price for the game option. Moreover, for any $t \in[0, T]$, the saddle-point $\left(\tau_{p}^{*}, \tau_{c}^{*}\right) \in \mathcal{F}_{T}^{t} \times \overline{\mathcal{F}}_{T}^{t}$ for the related Dynkin game (2.6) on $\mathcal{G}_{T}^{t} \times \overline{\mathcal{G}}_{T}^{t}$ is given by

$$
\tau_{p}^{*}=\inf \left\{u \in[t, T] ; \tilde{\Pi}_{u} \leq L_{u}\right\} \wedge T, \quad \tau_{c}^{*}=\inf \left\{u \in[\bar{\tau} \vee t, T] ; \tilde{\Pi}_{u} \geq U_{u}\right\} \wedge T .
$$

Proof. In view of (3.4), the present assumptions imply that $\widetilde{\Pi}_{t}$ is the value of the Dynkin game (3.6), by Proposition 3.12, with saddle-point $\left(\tau_{p}^{*}, \tau_{c}^{*}\right)$. Therefore, by Lemmas 3.5 and 3.6, $\Pi_{t}$ is the value of the Dynkin game associated with the game option on $\mathcal{G}_{T}^{t} \times \overline{\mathcal{G}}_{T}^{t}$, with saddlepoint $\left(\tau_{p}^{*}, \tau_{c}^{*}\right)$. Moreover, $\Pi$ is a $\mathbb{G}$-semimartingale, by Lemma 3.14(ii). To conclude the proof, it suffices to make use of the last statement in Theorem 3.8.

\section{Hedging in a Hazard Process Setup}

In the remaining part of this work, we examine in some detail the existence and basic properties of hedging strategies for defaultable game options in a hazard process setup. 


\subsection{Cost Process of a Hedging Strategy}

From now on, we will work under Assumption 3.13. Let thus $(\bar{\Theta}, M, K)$ denote a solution to $(\overline{\mathcal{E}})$ and let $\tilde{\Pi}$ and $\Pi$ be defined by (3.19). In particular, $\Pi$ is the arbitrage $\mathbb{Q}$-price for the game option (by Proposition 3.16) and the left-hand sides in (2.14) and (2.15) are equal to $\Pi_{0}$. Finally, recall that the $\mathbb{G}$-martingale $m$ is defined by (3.20).

Let us stress that some of the key arguments underlying the following result are classical, and they are already contained in Lepeltier and Maingueneau [19] (see, in particular, Theorem 11 therein). Proposition 4.1 can thus be seen as a natural extension of their results to the defaultable case, in which two filtrations are involved. It is notable that our assumptions are made relative to the filtration $\mathbb{F}$, whereas conclusions are drawn relative to the filtration $\mathbb{G}$.

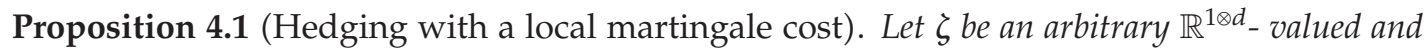
$\beta \widehat{X}$-integrable process. Then the following statements are valid.

(i) Let the process $\rho(\zeta)$ be given by $\rho_{0}(\zeta)=0$ and

$$
\beta_{t} d \rho_{t}(\zeta)=d m_{t}-\zeta_{t} d\left(\beta_{t} \hat{X}_{t}\right)
$$

Then $\left(\Pi_{0}, \zeta, \rho(\zeta), \tau_{c}^{*}\right)$ is an issuer hedge with $\mathbb{G}$-sigma (local, in case $\beta \widehat{X}$ and $\zeta$ are locally bounded) martingale cost.

(ii) Let the process $\bar{\rho}(\zeta)$ be given by $\bar{\rho}_{0}(\zeta)=0$ and

$$
\beta_{t} d \bar{\rho}_{t}(\zeta)=-d m_{t}-\zeta_{t} d\left(\beta_{t} \widehat{X}_{t}\right)
$$

Then $\left(-\Pi_{0}, \zeta, \bar{\rho}(\zeta), \tau_{p}^{*}\right)$ is a holder hedge with a $\mathbb{G}$-sigma martingale (local martingale, when $\beta \hat{\mathrm{X}}$ and $\zeta$ are locally bounded) cost process.

Recall that, according to our convention (see Section 1.2), the $\beta \hat{X}$-integrability of an $\mathbb{R}^{1 \otimes d}$-valued stochastic process $\zeta$ implies its $\mathbb{G}$-predictability. Note also that the equality $\bar{\rho}(-\zeta)=-\rho(\zeta)$ is valid for any process $\zeta$, since

$$
-\beta_{t} d \rho_{t}(\zeta)=-d m_{t}+\zeta_{t} d\left(\beta_{t} \widehat{X}_{t}\right)=-d m_{t}-\left(-\zeta_{t}\right) d\left(\beta_{t} \widehat{X}_{t}\right)
$$

Proof of Proposition 4.1. The arguments for a holder are essentially symmetrical to those for an issuer; we thus only prove part (i). By Lemma 3.14(i), the process $\rho(\zeta)$ is a $\mathbb{G}$-sigma martingale, and a $\mathbb{G}$-local martingale if $\beta \widehat{X}$ and $\zeta$ are locally bounded processes. For the ease of notation, we write $\rho=\rho(\zeta)$. Let $V$ denote the wealth process of the primary strategy $\left(\Pi_{0}, \zeta\right)$. By combining (2.9) with (4.1), we obtain $V_{0}=\Pi_{0}$ and, for every $t \in[0, T]$,

$$
d\left(\beta_{t} V_{t}\right)=\zeta_{t} d\left(\beta_{t} \widehat{X}_{t}\right)=d m_{t}-\beta_{t} d \rho_{t}
$$


and thus

$$
\beta_{t} V_{t}+\int_{0}^{t} \beta_{u} d \rho_{u}=m_{t}+\beta_{0}\left(\Pi_{0}-\widehat{\Pi}_{0}\right)=\beta_{t} \widehat{\Pi}_{t}+\int_{0}^{t \wedge \tau_{d}} \beta_{u} d K_{u}+\beta_{0}\left(\Pi_{0}-\widehat{\Pi}_{0}\right)
$$

where the second equality follows from (3.20). Recall that the stopping time $\tau_{c}^{*} \in \overline{\mathscr{F}}_{T}^{0}$ is given by (see Proposition 3.16)

$$
\tau_{c}^{*}=\inf \left\{t \in[\bar{\tau}, T] ; \tilde{\Pi}_{t} \geq U_{t}\right\} \wedge T
$$

In order to prove that the quadruplet $\left(\Pi_{0}, \zeta, \rho, \tau_{c}^{*}\right)$ is an issuer hedge for the game option, it is enough to show that one has for any $\tau_{p} \in \mathcal{F}_{T}^{0}$, with $\tau=\tau_{p} \wedge \tau_{c}^{*}$ (cf. (2.13)),

$$
\beta_{\tau} V_{\tau}+\int_{0}^{\tau} \beta_{u}\left(d \rho_{u}-d D_{u}\right) \geq 1_{\left\{\tau<\tau_{d}\right\}} \beta_{\tau}\left(1_{\left\{\tau=\tau_{p}<T\right\}} L_{\tau p}+1_{\left\{\tau<\tau_{p}\right\}} U_{\tau_{c}^{*}}+1_{\left\{\tau_{p}=\tau_{c}^{*}=T\right\}} \xi\right) .
$$

From the definition of $\tau_{c}^{*}$, the minimality conditions in $(\overline{\mathcal{\varepsilon}})$ and the continuity of $K^{-}$it follows that $K^{-}=0$ and thus $K \geq 0$ on $\left[0, \tau_{c}^{*}\right]$. Since $\tau \leq \tau_{c}^{*}$, (4.5) thus yields

$$
\beta_{\tau} V_{\tau}+\int_{0}^{\tau} \beta_{u}\left(d \rho_{u}-d D_{u}\right)=\beta_{\tau} \Pi_{\tau}+\int_{\left[0, \tau \wedge \tau_{d}\right]} \beta_{u} d K_{u} \geq \beta_{\tau} \Pi_{\tau}=1_{\left\{\tau<\tau_{d}\right\}} \beta_{\tau} \widetilde{\Pi}_{\tau}
$$

where, by $(\overline{\mathcal{E}})$, one has that

$$
\tilde{\Pi}_{\tau} \geq 1_{\{\tau<T\}} L_{\tau}+1_{\{\tau=T\}} \xi .
$$

In addition, by the definition of $\tau_{c}^{*}$, one has that $\tilde{\Pi}_{\tau_{c}^{*}} \geq U_{\tau_{c}^{*}}$ on the event $\left\{\tau_{c}^{*}<T\right\}$. It is now easy to see that (4.7) is satisfied and thus $\left(V_{0}, \zeta, \rho, \tau_{c}^{*}\right)$ is indeed an issuer hedge.

Remark 4.2. (i) The situation where $\rho$ can be made equal to zero by the choice of a suitable strategy $\zeta$ in Proposition 4.1 corresponds to a particular form of hedgeability of a game option in which an issuer and a holder are able to hedge all risks embedded in a defaultable game option. The case where $\rho \neq 0$ corresponds either to nonhedgeability of a game option or to the situation in which an issuer (or a holder) is able to hedge, but she prefers not to hedge all risks associated with a game option, for instance, she may be willing to take some directional bets regarding specific risks. For this reason, we decided not to postulate a priori that $\rho$ should be minimized in some sense as, for instance, in Schweizer [24].

(ii) It is possible to introduce the issuer trivial hedge $\left(\Pi_{0}, 0, \rho^{0}, \tau_{c}^{*}\right)$ (resp., the holder trivial hedge $\left.\left(-\Pi_{0}, 0,-\rho^{0}, \tau_{p}^{*}\right)\right)$ with the $\mathbb{G}$-local martingale cost

$$
\rho_{t}^{0}=\int_{0}^{t} \beta_{u}^{-1} d m_{u}, \quad t \in[0, T]
$$


Obviously, this hedge is of no practical interest, since it implicitly assumes that one is not interested in hedging any risks. The trivial hedge or, more precisely, the existence of any hedge is used in the proof of Proposition 4.3, however.

Let us now draw some conclusions from Lemma 2.13 and Proposition 4.1. In the context of specific (Cox-Ross-Rubinstein or Black-Scholes, say) models, analogous results can be found in Kifer [8]. Our main contribution here is an extension of these results to the present setup involving a reduction of filtration, as well as to a fairly general class of semimartingale models. We use here the notation ess min (instead of a more common symbol essinf) in order to emphasize that the respective bounds are in fact attained.

Proposition 4.3. Under the assumptions of Proposition 4.1, the following statements are valid.

(i) The equality $\Pi_{0}=$ ess min $\mho_{0}^{c}$ holds, so that $\Pi_{0}$ is the minimum of initial wealths of an issuer hedge with a $\mathbb{G}$-sigma martingale cost.

(ii) One has that $-\Pi_{0} \in \mho_{0}^{p}$. If, in addition, (2.5) holds then $\Pi_{0}=-e s s$ min $\mho_{0}^{p}$ and $-\Pi_{0}$ is the minimum of initial wealths of a holder hedge with a $\mathbb{G}$-sigma martingale cost.

(iii) The above statements are also valid with local martingale instead of sigma martingale therein.

Proof. (i) By applying Proposition 4.1 to the trivial hedge of Remark 4.2(ii), we get, in particular, that $\Pi_{0} \in \mathcal{V}_{0}^{c}$, where $\Pi_{0}$ is also equal to the $\mathbb{Q}$-value of the related Dynkin game, by Proposition 3.16. Therefore, the infimum is attained and one has equality, rather than inequality, in Lemma 2.13(i).

(ii) In view of (2.5) and Lemma 2.13(ii), the second claim can be proven in the same way as part (i).

(iii) This follows immediately from parts (i) and (ii), since the cost $\rho^{0}$ of the trivial hedge is a $\mathbb{G}$-local martingale.

Given our definition of hedging with a cost and the definition of $\Pi_{0}$, the fact that there exists a hedge with an initial wealth $\Pi_{0}$ and a $\mathbb{G}$-sigma martingale cost (or a local martingale cost, in suitable cases) is by no means surprising. The minimality statement establishes a connection between arbitrage prices and hedging in a general incomplete market. Let us conclude this section by mentioning that one could state analogous definitions and results regarding hedging strategies for a defaultable game option starting at any date $t \in[0, T]$.

\subsection{Risk Factors of a Defaultable Game Option}

Let $N^{d}=H-\Gamma \cdot \wedge \tau_{d}$ stand for the compensated default process. Under our standing assumption that the $\mathbb{F}$-hazard process $\Gamma$ of $\tau_{d}$ is a continuous and nondecreasing process (cf. Remark 3.2(ii)), the process $N^{d}$ is known to be a $\mathbb{G}$-martingale. Recall also that the avoidance property holds, in the sense that $\mathbb{Q}\left(\tau_{d}=\tau\right)=0$ for any $\mathbb{F}$-stopping time $\tau$ (cf. Remark 3.2(i)).

An analysis of hedging strategies in the next section hinges on the following lemma, which yields the risk decomposition of the discounted cumulative value process of a defaultable game option. More formally, the martingale component $m$ (cf. Remark 3.15) is represented in terms of the pure jump martingale $N^{d}$ and a real-valued $\mathbb{F}$-martingale $M$, which arise as the second component of a solution to the doubly reflected BSDE (3.7). Intuitively, the process $M$ models the pre-default risk associated with a defaultable game 
option, as opposed to the event risk, which is due to an unexpected occurrence of the default event, and which is modeled through the jump martingale $N^{d}$.

Lemma 4.4. The $\mathbb{G}$-martingale $m$ defined by (3.20) satisfies

$$
d m_{t}=1_{\left\{t \leq \tau_{d}\right\}} \beta_{t}\left(d M_{t}+Y_{t} d N_{t}^{d}\right)
$$

where the $\mathbb{F}$-predictable process $Y$ equals $Y_{t}=R_{t}-\widetilde{\Pi}_{t-}$.

Proof. Let us introduce the Doléans-Dade martingale (see, e.g., [29])

$$
\varepsilon_{t}=1_{\left\{t<\tau_{d}\right\}} e^{\Gamma_{t}}=1-\int_{0}^{t} \mathcal{E}_{u-} d N_{u}^{d}
$$

so that $\alpha_{t} \varepsilon_{t}=\beta_{t} 1_{\left\{t<\tau_{d}\right\}}$ and $\alpha_{t} \varepsilon_{t-}=\beta_{t} 1_{\left\{t \leq \tau_{d}\right\}}$. Then (cf. (3.19) and (3.20))

$$
d m_{t}=d\left(\beta_{t} \widehat{\Pi}_{t}\right)+1_{\left\{t \leq \tau_{d}\right\}} \beta_{t} d K_{t}=d\left(\varepsilon_{t} \alpha_{t} \tilde{\Pi}_{t}\right)+1_{\left\{t \leq \tau_{d}\right\}} \beta_{t} d K_{t}+\beta_{t} d D_{t}
$$

It may happen that the $\mathbb{F}$-semimartingale $\alpha \widetilde{\Pi}$ fails to be also a $\mathbb{G}$-semimartingale, so a direct application of the $(\mathbb{G}$-)integration by parts formula to $\mathcal{\varepsilon} \alpha \tilde{\Pi}$ is not possible. However, by Lemma A.1(iv), the process $\alpha \tilde{\Pi}$ stopped at $\tau_{d}$ is a $\mathbb{G}$-semimartingale. It is also clear that $\varepsilon \alpha \tilde{\Pi}=\varepsilon \alpha_{\cdot \wedge \tau_{d}} \tilde{\Pi}_{\cdot \wedge \tau_{d}}$. Hence by applying the integration by parts formula to $\varepsilon \alpha_{\cdot \wedge \tau_{d}} \tilde{\Pi}_{\cdot \wedge \tau_{d}}$, we obtain

$$
d\left(\varepsilon_{t} \alpha_{t \wedge \tau_{d}} \tilde{\Pi}_{t \wedge \tau_{d}}\right)=\varepsilon_{t-}\left(d\left(\alpha_{t \wedge \tau_{d}} \tilde{\Pi}_{t \wedge \tau_{d}}\right)-\alpha_{t} \tilde{\Pi}_{t-} d N_{t}^{d}\right)+d\left[\varepsilon, \alpha_{\cdot \wedge \tau_{d}} \tilde{\Pi}_{\cdot \wedge \tau_{d}}\right]_{t^{\prime}}
$$

where, in addition, one has that $\left[\mathcal{E}, \alpha_{\cdot \wedge \tau_{d}} \tilde{\Pi}_{\wedge \tau_{d}}\right]_{t}=-e^{\Gamma_{\tau d}} \alpha_{\tau_{d}} \Delta \widetilde{\Pi}_{\tau_{d}} H_{t}$. Using the avoidance property of Remark 3.2(i), formula (3.22), and the assumptions that the coupon process $C$ is $\mathbb{F}$-predictable and the hazard process $\Gamma$ is continuous (so that $\Delta C_{\tau_{d}}=\Delta \Gamma_{\tau_{d}}=0$ ), we obtain the equality $\Delta \widetilde{\Pi}_{\tau_{d}}=0$. Using (3.22), we next deduce from (4.13) that

$$
\begin{aligned}
d m_{t} & =\varepsilon_{t-}\left(d\left(\alpha_{t \wedge \tau_{d}} \tilde{\Pi}_{t \wedge \tau_{d}}\right)-\alpha_{t} \tilde{\Pi}_{t-} d N_{t}^{d}\right)+1_{\left\{t \leq \tau_{d}\right\}} \beta_{t} d K_{t}+\beta_{t} d D_{t} \\
& =1_{\left\{t \leq \tau_{d}\right\}} \beta_{t}\left(-d K_{t}-d C_{t}-R_{t} d \Gamma_{t}+d M_{t}-\tilde{\Pi}_{t-} d N_{t}^{d}\right)+1_{\left\{t \leq \tau_{d}\right\}} \beta_{t} d K_{t}+\beta_{t} d D_{t} \\
& =1_{\left\{t \leq \tau_{d}\right\}} \beta_{t}\left(-d C_{t}-R_{t} d \Gamma_{t}+d M_{t}-\tilde{\Pi}_{t-} d N_{t}^{d}\right)+\beta_{t} d D_{t} .
\end{aligned}
$$

Using (2.3) and the equality $\Delta C_{\tau_{d}}=0$, we finally arrive at the formula

$$
d m_{t}=1_{\left\{t \leq \tau_{d}\right\}} \beta_{t}\left(d M_{t}+\left(R_{t}-\tilde{\Pi}_{t-}\right) d N_{t}^{d}\right)
$$

which is the required result. 


\subsection{Hedging of Risk Factors}

In order to study nontrivial cases of hedging strategies for a defaultable game option in the general setup of this paper, we need to impose more assumptions on prices of primary traded assets. Since we are working in a fairly general framework, we will be able to provide only general results concerning hedging strategies. The interested reader is referred to the followup papers $[4,9]$ for a more detailed analysis of assumptions made in this section and particular examples.

First, we recall that the ex-dividend price $X$ of primary risky assets satisfies $X_{t}=(1-$ $\left.H_{t}\right) \tilde{X}_{t}$, for every $t \in[0, T]$, where the $\mathbb{R}^{d}$-valued, $\mathbb{F}$-adapted process $\tilde{X}$ formally represents the pre-default value of $X$. We thus assume, by convention, that any residual value of the primary asset at $\tau_{d}$ is embedded in the recovery part of the dividend process for $X$. We denote by $R$ an $\mathbb{R}^{d}$-valued and $\mathbb{F}$-predictable process, which is aimed to represent the recovery processes of primary risky assets. Inspired by decomposition (4.11) of Lemma 4.4, we make also the following natural postulate regarding the behavior of the cumulative price process $\hat{X}$ stopped at $\tau_{d} \wedge T$.

Assumption 4.5. The dynamics under $\mathbb{Q}$ of the cumulative price process $\hat{X}$ of primary risky assets are, for every $t \in\left[0, T \wedge \tau_{d}\right]$,

$$
d\left(\beta_{t} \widehat{X}_{t}\right)=\beta_{t}\left(d \widehat{M}_{t}+\widehat{Y}_{t} d N_{t}^{d}\right)
$$

for some $\mathbb{R}^{d}$-valued $\mathbb{F}$-martingale $\widehat{M}$, where the $\mathbb{R}^{d}$-valued, $\mathbb{F}$-predictable process $\widehat{Y}$ is given by the equality $\widehat{Y}_{t}=\mathcal{R}_{t}-\tilde{X}_{t-}$ for every $t \in[0, T]$.

By inserting (4.11) and (4.17) into (4.1), we obtain, for every $t \in\left[0, T \wedge \tau_{d}\right]$,

$$
d \rho_{t}(\zeta)=d M_{t}-\zeta_{t} d \widehat{M}_{t}+\left(Y_{t}-\zeta_{t} \widehat{Y}_{t}\right) d N_{t}^{d}
$$

At this stage, we were only able to separate the two principal components of the cost process that correspond to pre-default and default event risks, respectively, where the pre-default risk is now modeled by the $\mathbb{F}$-martingales $M$ and $\widehat{M}$ associated with a game option and primary traded assets, respectively.

Remark 4.6. In what follows, we will only be interested in hedging on the random interval $\left[0, \tau_{d} \wedge T\right]$. Therefore, without loss of generality, we may and do assume that $\zeta$ is $\mathbb{F}$-predictable (see Lemma A.2(ii)). This means that the reduction of filtration method can also be applied to hedging of a defaultable game option, and not only to its valuation as was already shown in Section 3.2.

Within the present framework, the event risk factor is common for all traded primary and derivative assets. Therefore, in the next step, we are going to get a closer look on pre-default risks of traded and derivative assets. To this end, we make a further standing assumption, in which the concept of the systematic risk factor (also known as the market risk factor) is introduced. 
Assumption 4.7. We are given an $\mathbb{R}^{q}$-valued $\mathbb{F}$-martingale, denoted by $N$, which is aimed to represent the systematic risk factor for the underlying market model. We postulate that the $\mathbb{F}$ martingales $M$ and $\widehat{M}$ of (4.11) and (4.17) satisfy the following decompositions, for every $t \in[0, T]$,

$$
d M_{t}=Z_{t} d N_{t}+d n_{t}, \quad d \widehat{M}_{t}=\widehat{Z}_{t} d N_{t}+d \widehat{n}_{t}
$$

where $Z$ (resp., $\hat{Z}$ ) is some $\mathbb{F}$-adapted, $\mathbb{R}^{1 \otimes q}$-valued (resp., $\mathbb{R}^{d \otimes q}$-valued), $N$-integrable processes and $n$ (resp., $\widehat{n}$ ) is a real-valued (resp., $\mathbb{R}^{d}$-valued) $\mathbb{F}$-martingale.

It is natural to refer to $\mathbb{F}$-martingales $n$ and $\hat{n}$ appearing in Assumption 4.7 as idiosyncratic risk factors associated with a defaultable game option and primary traded assets, respectively. In this context, we find it convenient to refer to $N^{d}$ as the event risk factor.

Remark 4.8. A specification of the systematic risk factor $N$ depends on a particular market model and on a problem at hand, so that it is not possible to make it more explicit in the abstract setup considered here. As it will become apparent in the sequel, the idiosyncratic risk factors are expected to be in some sense orthogonal to the systematic risk factor. For this reason, one cannot simply make $Z$ and $\widehat{Z}$ to vanish in (4.19). Once again, for more information on particular models, we refer to $[4,9]$ (see also Remark 3.10).

Let us denote $\mathcal{N}=\left[\begin{array}{c}N \\ N^{d}\end{array}\right]$ and let $[Z, Y]$ stand for the concatenation of $Z$ and $Y$. The next lemma is an immediate consequence of (4.18) and (4.19). The idea behind formula (4.20) is the separation of risk factors $N, N^{d}, n$, and $\widehat{n}$ in the dynamics of the cost process of a trading strategy.

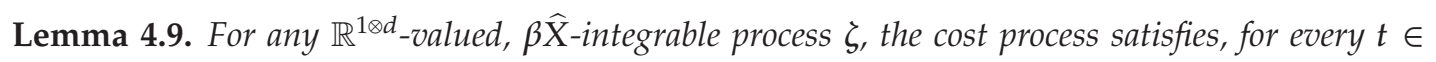
$\left[0, T \wedge \tau_{d}\right]$

$$
d \rho_{t}(\zeta)=\left(\left[Z_{t}, Y_{t}\right]-\zeta_{t}\left[\widehat{Z}_{t}, \widehat{Y}_{t}\right]\right) d \mathcal{N}_{t}+d n_{t}-\zeta_{t} d \widehat{n}_{t}
$$

Example 4.10. To provide some intuition underpinning the present setup, let us first consider a situation where the perfect hedgeability of risks can be achieved, at least in principle. Let us set $q=1$ and we take $d M_{t}=Z_{t} d N_{t}$, so that $n$ vanishes. For $d=2$, we further postulate that $\widehat{n}=0$ and

$$
d \widehat{M}_{t}=d\left[\begin{array}{c}
\widehat{M}_{t}^{1} \\
\widehat{M}_{t}^{2}
\end{array}\right]=\left[\begin{array}{c}
\widehat{Z}_{t}^{1} \\
\widehat{Z}_{t}^{2}
\end{array}\right] d M_{t}
$$

or, equivalently, that for $i=1,2$

$$
d\left(\beta_{t} \widehat{X}_{t}^{i}\right)=\beta_{t}\left(\widehat{Z}_{t}^{i} d M_{t}+\widehat{Y}_{t}^{i} d N_{t}^{d}\right)
$$


Assume that there exists a $\beta \widehat{X}$-integrable process $\zeta$ solving the equation

$$
\zeta_{t}\left[\widehat{Z}_{t}, \widehat{Y}_{t}\right]=\left[\zeta_{t}^{1}, \zeta_{t}^{2}\right]\left[\begin{array}{cc}
\hat{Z}_{t}^{1} & \widehat{Y}_{t}^{1} \\
\widehat{Z}_{t}^{2} & \widehat{Y}_{t}^{2}
\end{array}\right]=\left[Z_{t}, Y_{t}\right] .
$$

Then it follows from (4.20) (or (4.18)) that the cost process $\rho(\zeta)$ vanishes and thus the strategy $\zeta$ (resp., $-\zeta$ ) is an issuer's (resp., holder's) superhedge for a defaultable game option, in the sense of Definition 2.10. Note that the first (resp., the second) equation in formula (4.23) is used to eliminate the pre-default risk (resp., the event risk). As was expected, the strategy $\zeta$ obtained by solving (4.23) is $\mathbb{F}$-predictable (cf. Remark 4.6).

Remark 4.11. In [4], we further specify the setup of Example 4.10, by examining the exact replication of a convertible bond with the equity and the credit default swap on the underlying credit name in an equity-to-credit intensity-based model, in which the systematic risk factor is modeled by the Brownian motion driving the equity value and all processes appearing in (4.23) can be computed explicitly.

In the foregoing result, we examine two typical situations regarding the partial hedgeability of risk factors when superhedging is either not possible or not desirable. The case considered in part (i) refers to elimination of event and systematic risks. In contrast, part (ii) deals with hedging of the systematic risk only. Of course, it is also possible to hedge the event risk only, but we do not formulate here the corresponding result. Since the proof of the lemma follows easily from (4.20), it is omitted.

Lemma 4.12 (Hedging of risk factors). (i) Assume that the equation $[Z, Y]=\zeta[\widehat{Z}, \hat{Y}]$ admits a $\beta \widehat{X}$-integrable solution $\widehat{\zeta}$ on $\left[0, T \wedge \tau_{d}\right]$. Then the cost process $\widehat{\rho}=\rho(\widehat{\zeta})$ satisfies, for every $t \in\left[0, T \wedge \tau_{d}\right]$,

$$
d \widehat{\rho}_{t}=d n_{t}-\widehat{\zeta}_{t} d \widehat{n}_{t}
$$

(ii) Assume that the equation $Z=\zeta \widehat{Z}$ admits a $\beta \widehat{X}$-integrable solution $\zeta$ on $\left[0, T \wedge \tau_{d}\right]$. Then the dynamics of the cost process $\check{\rho}=\rho(\breve{\zeta})$ are, for every $t \in\left[0, T \wedge \tau_{d}\right]$,

$$
d \check{\rho}_{t}=\left(Y_{t}-\breve{\zeta}_{t} \widehat{Y}_{t}\right) d N_{t}^{d}+d n_{t}-\check{\zeta}_{t} d \widehat{n}_{t} .
$$

Part (i) in Lemma 4.12 corresponds to the case where the common risks (systematic and event) can be completely eliminated. In contrast, part (ii) refers either to the case of unhedgeable event risk (e.g., when $\hat{Y}=0$ in dynamics (4.17)) or to the situation when the issuer (or holder) is not willing to hedge that risk.

As was already mentioned, practically useful decompositions of $M$ and $\widehat{M}$ will depend on a particular model for the primary market, as well as on the game option under study. In an abstract setup considered here, they may be formally deduced from martingale representation theorems with orthogonal components.

Let thus $\mathfrak{d}^{2}$ stand for the class of real-valued $\mathbb{F}$-martingales with integrable quadratic variation over $[0, T]$ or, by a slight abuse of notation, the class of vector-valued processes with mutually strongly orthogonal components in $\mathfrak{l}^{2}$. It is worth recalling here that an $\mathbb{F}$ martingale stopped at $\tau_{d}$ is also a $\mathbb{G}$-local martingale, by virtue of Lemma A.1(iii). 
The Galtchouk-Kunita-Watanabe (GKW) decomposition of $M$ and $\widehat{M}$ with respect to $N$ and the filtration $\mathbb{F}$ (see, e.g., Protter [16, Section IV.3, Corollary 1]) thus yields the decompositions (4.19) of $M$ and $\widehat{M}$ with $n$ and $\widehat{n}$ strongly orthogonal to $N$ in $\mathscr{L}^{2}$. Since $N$ is meant to represent the systematic risk factor, we may and do assume, without loss of generality, that the idiosyncratic risk factors $n$ and $\hat{n}$ are also mutually strongly orthogonal.

The following proposition justifies the informal statement that the strategy $\widehat{\zeta}$ (resp., $\breve{\zeta}$ ) hedges the risk factor $\mathcal{N}$ (resp., $N$ ). We use hereafter the standard symbol $[\cdot, \cdot]$ to denote the square bracket between $\mathbb{G}$-semimartingales.

Proposition 4.13 (Orthogonality of risk factors). Assume that the processes $N, n$, and $\hat{n}$ in decompositions (4.19) of $M$ and $\widehat{M}$ are mutually strongly orthogonal in $\mathfrak{L}^{2}$.

(i) Under assumptions of Lemma $4.12(i)$, the processes $\hat{\rho}$ and $\mathcal{N}_{\wedge} \tau_{d}$ are orthogonal in $\mathbb{G}$, in the sense that $\left[\hat{\rho}, N_{\cdot \wedge \tau_{d}}\right]$ is a $\mathbb{G}$-sigma martingale (and a $\mathbb{G}$-local martingale if $\widehat{\zeta}$ is locally bounded).

(ii) Under assumptions of Lemma $4.12(i i)$, the processes $\check{\rho}$ and $N_{. \wedge \tau_{d}}$ are orthogonal in $\mathbb{G}$, in the sense that $\left[\check{\rho}, N_{\cdot \wedge \tau_{d}}\right]$ is a $\mathbb{G}$-sigma martingale (and a $\mathbb{G}$-local martingale if $\breve{\zeta}, R$, and $\mathcal{R}$ are locally bounded processes).

Proof. We first note that $n_{\cdot \wedge \tau_{d}}$ and $N_{\cdot \wedge \tau_{d}}$ are $\mathbb{G}$-local martingales, by Lemma A.1(iii). Since $n$ is strongly orthogonal to $N$ in $\mathscr{\ell}^{2}$, the process $\left[n_{\cdot \wedge \tau_{d}}, N_{\cdot \wedge \tau_{d}}\right]$ is a $\mathbb{G}$-local martingale, as an $\mathbb{F}$-local martingale stopped at $\tau_{d}$ (cf. Lemma A.1(iii)). Furthermore, by Lemma A.6, $\left[n_{\cdot \wedge \tau_{d}}, N^{d}\right]$ is a $\mathbb{G}$ local martingale. We conclude that $\left[n \cdot \wedge \tau_{d}, \mathcal{N}_{\cdot \wedge \tau_{d}}\right]$ is a $\mathbb{G}$-local martingale. So are also $\left[\widehat{n}, N \cdot \wedge \tau_{d}\right]$ and $\left[\widehat{n}, \mathcal{N}_{\wedge} \wedge \tau_{d}\right]$, since the integral $\int_{0} \beta_{t} d \widehat{n}_{t}$ is strongly orthogonal to $\mathcal{N}_{\wedge} \wedge \tau_{d}$. Furthermore, by Lemma A.6, $\left[N_{\cdot \wedge \tau_{d},}, N^{d}\right]$ is a $\mathbb{G}$-local martingale.

Using (4.24), we conclude for part (i) that $\left[\hat{\rho}, \mathcal{N}_{\wedge} \wedge \tau_{d}\right]$ is a $\mathbb{G}$-sigma martingale and thus it follows a $\mathbb{G}$-local martingale if $\hat{\zeta}$ is a locally bounded process.

For part (ii), we conclude in view of $(4.25)$ that $\left[\check{\rho}, N_{\cdot \wedge \tau_{d}}\right]$ is a $\mathbb{G}$-sigma martingale and thus a $\mathbb{G}$-local martingale if $\breve{\zeta}, R$ and $\mathcal{R}$ are locally bounded processes.

\subsection{Hedging with Orthogonal Cost}

Before concluding this work, let us examine briefly an alternative approach to hedging a defaultable game option, which is formally defined as the problem of finding a strategy $\zeta$ that makes the cost process $\mathbb{G}$-orthogonal under a given risk-neutral probability measure $\mathbb{Q}$ to a predetermined $\mathbb{R}^{q}$-valued $\mathbb{G}$-local martingale $\bar{N}$ where, without loss of generality, the process $\bar{N}$ is assumed to be stopped at $\tau_{d}$. In reference to Proposition 4.13 , by the $\mathbb{G}$-orthogonality, we mean here that $[\rho, \bar{N}]$ is a $\mathbb{G}$-local martingale under $\mathbb{Q}$.

Remark 4.14. In the financial interpretation, the process $\bar{N}$ may represent the wealth processes of some preexisting portfolios, rather than risk factors as in Sections 4.2 and 4.3. Admittedly, we consider here a reduced concept of hedging, at least from the theoretical perspective. It is possible to argue, however, that this approach may be of practical relevance, since some kind of a relative hedging (as opposed to replication or superhedging) is a common market practice.

For the purpose of this section, the process $m$ arising in (4.1) may be defined either by (3.20), in reference to a solution of a related doubly reflected BSDE with respect to the filtration $\mathbb{F}$ or, more generally (cf. Remark 3.15), as the $\mathbb{G}$-local martingale component of the discounted cumulative $\mathbb{Q}$-value process $\beta \widehat{\Pi}$ of a game option, provided that $\beta \widehat{\Pi}$ is a $\mathbb{G}$-special semimartingale. 
In the following proposition we denote (whenever well-defined)

$$
\operatorname{Cov}_{t}\left(d X_{t}, d Y_{t}\right)=\lim _{h \rightarrow 0} h^{-1} \operatorname{Cov}\left(X_{t+h}-X_{t}, Y_{t+h}-Y_{t} \mid \mathcal{G}_{t}\right)
$$

and $\operatorname{Var}_{t}\left(d X_{t}\right)=\operatorname{Cov}_{t}\left(d X_{t}, d X_{t}\right)$.

Proposition 4.15 (Hedging with orthogonal cost). Assume that $\widehat{X}$ admits the decomposition, for every $t \in\left[0, T \wedge \tau_{d}\right]$,

$$
d\left(\beta_{t} \widehat{X}_{t}\right)=\beta_{t} \bar{Z}_{t} d \bar{N}_{t}+\beta_{t} d \bar{n}_{t}
$$

with $\bar{n}$ and $\bar{N}$ orthogonal in $\mathbb{G}$ and an $\mathbb{R}^{d \otimes q_{\text {-valued, }}} \bar{N}$-integrable process $\bar{Z}$, which is left-invertible on $\left[0, T \wedge \tau_{d}\right]$. Let us set, for every $t \in\left[0, T \wedge \tau_{d}\right]$,

$$
\zeta_{t}=\operatorname{Cov}_{t}\left(d m_{t}, \beta_{t} d \bar{N}_{t}\right) \operatorname{Var}_{t}\left(\beta_{t} d \bar{N}_{t}\right)^{-1} \Lambda_{t}
$$

where $\Lambda$ is the left inverse of the transpose of $\bar{Z}$ on $\left[0, T \wedge \tau_{d}\right]$. Then the cost process $\rho(\zeta)$ is orthogonal to $\bar{N}$ in $\mathbb{G}$.

Proof. By combining (4.1) with (4.27), we obtain

$$
\beta_{t} d \rho_{t}(\zeta)=d m_{t}-\zeta_{t} \beta_{t} \bar{Z}_{t} d \bar{N}_{t}-\beta_{t} \zeta_{t} d \bar{n}_{t}
$$

Therefore, in order to have the cost $\rho$ orthogonal to $\bar{N}$ in $\mathbb{G}$, it suffices to select a strategy $\zeta$ for which $m-\int_{0} \beta_{t} \zeta_{t} \bar{Z}_{t} d \bar{N}_{t}$ is $\mathbb{G}$-orthogonal to $\bar{N}$. Relying on the multilinear regression formula, this can be achieved by setting $\zeta$ as in (4.28).

The problem of hedging a defaultable game option with respect to $\bar{N}$ can thus be solved, at least formally, provided that one can find a decomposition (4.27) with the required properties. Such a decomposition can be obtained as the GKW decomposition of $\beta \hat{X}$ in $\mathbb{G}$ with respect to $\bar{N}$, provided that the related matrix $\bar{Z}$ is left-invertible on $\left[0, T \wedge \tau_{d}\right]$. It is then natural to conjecture that the strategy given by (4.28) for $\bar{N}=\mathcal{N}_{\wedge \wedge \tau_{d}}\left(\operatorname{resp} ., \bar{N}=N_{\cdot \wedge \tau_{d}}\right.$ ) will coincide with the strategy $\widehat{\zeta}$ (resp., $\breve{\zeta}$ ) of Proposition 4.13 .

The following result examines the special case when $\bar{n}=0$ in (4.27). As can be seen from formula (4.31) below, this corresponds to the assumption that the process $\bar{N}$ represents the wealth processes of some portfolios of primary traded assets.

Corollary 4.16 (Min-variance hedging). Assume that $\bar{n}=0$ in (4.27) and $\bar{Z}$ is left-invertible on $\left[0, T \wedge \tau_{d}\right]$. Then the strategy $\zeta$ of Proposition 4.15 becomes

$$
\zeta_{t}=\operatorname{Cov}_{t}\left(d m_{t}, d\left(\beta_{t} \widehat{X}_{t}\right)\right) \operatorname{Var}_{t}\left(d\left(\beta_{t} \widehat{X}_{t}\right)\right)^{-1}
$$


Proof. Under the present assumptions, we obtain from (4.27)

$$
\beta_{t} d \bar{N}_{t}=\Lambda_{t} d\left(\beta_{t} \hat{X}_{t}\right), \quad t \in\left[0, T \wedge \tau_{d}\right]
$$

Consequently, formula (4.28) reduces to

$$
\zeta_{t}=\operatorname{Cov}_{t}\left(d m_{t}, \Lambda_{t} d\left(\beta_{t} \widehat{X}_{t}\right)\right) \operatorname{Var}_{t}\left(\Lambda_{t} d\left(\beta_{t} \widehat{X}_{t}\right)\right)^{-1} \Lambda_{t}
$$

and this in turn yields (4.31).

We recognize here a strategy, which is known to arise in the context of the minvariance hedging in incomplete markets. In the present setup, it was not derived by suitable optimization arguments, however, but obtained by simply postulating that the cost process should be orthogonal to prices of primary assets under the preselected risk-neutral probability measure $\mathbb{Q}$. It should be noted that in the context of a game (or even American) option, the min-variance hedging approach should also incorporate optimization with respect to exercise times. This would lead to the optimization problem of the form (from the issuer's perspective, cf. $(2.11))$

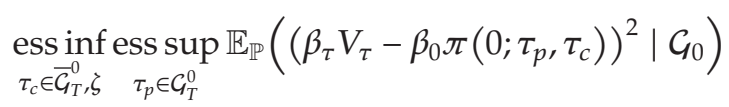

for a given level $V_{0}$ of the initial wealth, where $\tau=\tau_{c} \wedge \tau_{p} \wedge \tau_{d}$ and where the expectation is taken under the statistical probability measure $\mathbb{P}$. In a discrete-time setup, this problem was recently proposed and solved by Dolinsky and Kifer [36], who have also observed that the corresponding problem in a continuous-time framework is very difficult to deal with. Whether such an approach in continuous time is amenable to mathematical and practical solution is indeed far from trivial, so it is left as an open challenging problem.

\section{Appendix}

\section{A. Auxiliary Lemmas}

Recall that an $\mathbb{F}$-pseudo-stopping time $\tau$ is a random time such that $\mathbb{E}_{\mathbb{Q}} M_{\tau}=\mathbb{E}_{\mathbb{Q}} M_{0}$ for every bounded $\mathbb{F}$-martingale $M$ (see Nikeghbali and Yor [28, Remark 1]).

We work throughout under the standing Assumption 3.1.

Lemma A.1. (i) $G$ is a nonincreasing process.

(ii) The $\mathbb{G}$-stopping time $\tau_{d}$ is an $\mathbb{F}$-pseudo-stopping time.

(iii) Any $\mathbb{F}$-local martingale stopped at $\tau_{d}$ is a $\mathbb{G}$-local martingale.

(iv) Any $\mathbb{F}$-semimartingale stopped at $\tau_{d}$ is a $\mathbb{G}$-semimartingale.

(v) The integral process of a continuous integrand with respect to an $\mathbb{F}$-martingale stopped at $\tau_{d}$ is a $\mathbb{G}$-local martingale.

Proof. Since $G$ is a continuous supermartingale, it admits the Doob-Meyer decomposition $G=M-A$ with a continuous martingale component $M$ [15, page 44, Lemma 4.24]. Hence 
$M$ is in fact constant, as a continuous martingale with finite variation, and thus (i) holds. By [28, Theorem 4.5], (i) implies (ii) (note that the continuity of the filtration $\mathbb{F}$ is only used for the converse in [28, Theorem 4.5]). By [28, Theorem 4.4], (ii) implies (iii), which also immediately yields (iv). For (v), we note that an $\mathbb{F}$-martingale stopped at $\tau_{d}$ is a $\mathbb{G}$-local martingale, by virtue of (iii). The integral process of a continuous (hence predictable and locally bounded) integrand, with respect to an $\mathbb{F}$-martingale stopped at $\tau_{d}$, is thus a $\mathbb{G}$-local martingale (cf. Remark 2.2).

We recall the following well-known results. We refer the interested reader to Bielecki and Rutkowski [29, Lemma 5.1.2(ii) and Corollary 5.1.2] for (i) and Dellacherie et al. [37, page 186, Section 75] for (ii) (see also Proposition 9.12 of Nikeghbali [38]).

Lemma A.2. (i) Let $\chi$ be a $\mathcal{G}_{\infty_{\infty}}$-measurable random variable. For any $t \in \mathbb{R}_{+}$such that one of the members of the following equality is well defined in $\overline{\mathbb{R}}$ (e.g., $x$ bounded from one side), the other one is well defined as well and one has that

$$
1_{\left\{t<\tau_{d}\right\}} \mathbb{E}_{\mathbb{Q}}\left(X \mid \mathcal{G}_{t}\right)=1_{\left\{t<\tau_{d}\right\}} e^{\Gamma_{t}} \mathbb{E}_{\mathbb{Q}}\left(1_{\left\{t<\tau_{d}\right\}} \mathcal{X} \mid \mathcal{F}_{t}\right) .
$$

In particular, if $X$ is $\mathcal{G}_{t}$-measurable then $1_{\left\{t<\tau_{d}\right\}} \mathcal{X}=1_{\left\{t<\tau_{d}\right\}} \tilde{X}$ where $\tilde{X}=e^{\Gamma_{t}} \mathbb{E}_{\mathbb{Q}}\left(1_{\left\{t<\tau_{d}\right\}} \mid \mathcal{F}_{t}\right)$ is an $\mathcal{F}_{t}$-measurable random variable. So for any $\mathbb{G}$-adapted process $Y$ over $[0, T]$, there exists an $\mathbb{F}$-adapted process $\tilde{Y}$ over $[0, T]$ such that

$$
1_{\left\{t<\tau_{d}\right\}} Y_{t}=1_{\left\{t<\tau_{d}\right\}} \tilde{Y}_{t}, \quad t \in[0, T]
$$

(ii) For any $\mathbb{G}$-predictable process $Y$ over $[0, T]$, there exists an $\mathbb{F}$-predictable process $\tilde{Y}$ over $[0, T]$ such that

$$
1_{\left\{t \leq \tau_{d}\right\}} Y_{t}=1_{\left\{t \leq \tau_{d}\right\}} \tilde{Y}_{t}, \quad t \in[0, T]
$$

Remark A.3. In the $\mathbb{G}$-predictable case, the process $\tilde{Y}$ satisfying (A.3) is uniquely defined under Assumption 3.1, by [37, page 186].

For any $t \in[0, T]$, we denote by $\mathcal{F}_{T}^{t}$ the set of all $\mathbb{F}$-stopping times with values in $[t, T]$. Also, given a stopping time $\bar{\tau} \in \mathcal{F}_{T}^{0}$ let $\overline{\mathscr{F}}_{T}^{t}$ stand for the class $\left\{\tau \in \mathcal{F}_{T}^{t} ; \tau \geq \bar{\tau}\right\}$. The following result examines the relevant properties of these classes of stopping times.

Lemma A.4. (i) If $\tau \in \mathcal{G}_{T}^{t}$ for some $t \in[0, T]$ then there exists $\tilde{\tau} \in \mathcal{F}_{T}^{t}$ such that $\tau \wedge \tau_{d}=\tilde{\tau} \wedge \tau_{d}$. Moreover, if $\bar{\tau} \in \mathcal{G}_{T}^{0}$ and if $\tau \in \overline{\mathcal{G}}_{T}^{t}$ for some $t \in[0, T]$ then one has $\tilde{\tau} \wedge \tau_{d} \geq \bar{\tau} \wedge \tau_{d}$.

(ii) If $\bar{\tau} \in \mathcal{F}_{T}^{0}$ and $\tau \in \overline{\mathcal{G}}_{T}^{t}$ for some $t \in[0, T]$ then there exists $\tilde{\tau} \in \overline{\mathcal{F}}_{T}^{t}$ such that $\tau \wedge \tau_{d}=\tilde{\tau} \wedge \tau_{d}$.

Proof. Since $\tau$ is a $\mathbb{G}$-stopping time, by [37, page 186, Section 75$]$ there exists an $\mathbb{F}$-stopping time $\widehat{\tau}$ such that $\tau \wedge \tau_{d}=\widehat{\tau} \wedge \tau_{d}$. Moreover, since $\tau \in \mathcal{G}_{T}^{t}$, one has

$$
\tau \wedge \tau_{d}=(\tau \vee t) \wedge \tau_{d}=\left(\tau \wedge \tau_{d}\right) \vee\left(t \wedge \tau_{d}\right)=\left(\widehat{\tau} \wedge \tau_{d}\right) \vee\left(t \wedge \tau_{d}\right)=(\widehat{\tau} \vee t) \wedge \tau_{d}
$$


so that we may take $\tilde{\tau}=\widehat{\tau} \vee t \in \mathcal{F}_{T}^{t}$. Moreover, if $\tau \wedge \tau_{d} \geq \bar{\tau} \wedge \tau_{d}$ for some stopping time $\bar{\tau} \in \mathcal{G}_{T}^{0}$, then we also have that $\tilde{\tau} \wedge \tau_{d}=\tau \wedge \tau_{d} \geq \bar{\tau} \wedge \tau_{d}$, which proves (i).

For (ii), let $\breve{\tau} \in \mathcal{F}_{T}^{t}$ be such that $\tau \wedge \tau_{d}=\breve{\tau} \wedge \tau_{d}$, by (i). Assuming that $\bar{\tau} \in \mathcal{F}_{T}^{0}$, one has that $\tilde{\tau}=\breve{\tau} \vee \bar{\tau} \in \overline{\mathscr{F}}_{T}^{t}$. So

$$
\tilde{\tau} \wedge \tau_{d}=(\breve{\tau} \vee \bar{\tau}) \wedge \tau_{d}=\left(\breve{\tau} \wedge \tau_{d}\right) \vee\left(\bar{\tau} \wedge \tau_{d}\right)=\breve{\tau} \wedge \tau_{d}=\tau \wedge \tau_{d}
$$

where the third equality holds, since $\tau \in \overline{\mathcal{G}}_{T}^{t}$ implies that $\breve{\tau} \wedge \tau_{d} \geq \bar{\tau} \wedge \tau_{d}$, by (i).

Let us recall that for any $\mathbb{F}$-stopping time $\tau$ one has that (formula (A.6) can be found, e.g., in Dellacherie [39, T47])

$$
\mathbb{Q}\left(\tau_{d}>\tau \mid \mathcal{F}_{\tau}\right)=e^{-\Gamma_{\tau}} .
$$

The following lemma is of independent interest.

Lemma A.5. Assume that $\tau \in \mathcal{F}_{T}^{t}$ for some $t \in[0, T]$. Then one has the following.

(i) For any $\boldsymbol{F}_{\tau}$-measurable random variable $x$ such that at least one side of the following equality is well defined in $\overline{\mathbb{R}}$ (e.g., $x$ bounded from one side), the other one is also well defined and one has

$$
\mathbb{E}_{\mathbb{Q}}\left(1_{\left\{\tau<\tau_{d}\right\}} X \mid \mathcal{G}_{t}\right)=1_{\left\{t<\tau_{d}\right\}} e^{\Gamma_{t}} \mathbb{E}_{\mathbb{Q}}\left(e^{-\Gamma_{\tau}} X \mid \mathcal{F}_{t}\right) .
$$

(ii) For any $\mathbb{F}$-predictable process $Z$ such that at least one side of the following equality is well defined in $\overline{\mathbb{R}}$ (e.g., $Z$ is bounded from one side), the other one is also well defined and one has

$$
\mathbb{E}_{\mathbb{Q}}\left(1_{\left\{t<\tau_{d} \leq \tau\right\}} Z_{\tau_{d}} \mid \mathcal{G}_{t}\right)=1_{\left\{t<\tau_{d}\right\}} e^{\Gamma_{t}} \mathbb{E}_{\mathbb{Q}}\left(\int_{t}^{\tau} Z_{u} e^{-\Gamma_{u}} d \Gamma_{u} \mid \mathcal{F}_{t}\right)
$$

(iii) For any finite variation $\mathbb{F}$-predictable process $A$ such that at least one side of the following equality is well defined in $\overline{\mathbb{R}}$ (e.g., the variation of $A$ over $[0, T]$ is bounded from one side), the other one is also well defined and one has

$$
\mathbb{E}_{\mathbb{Q}}\left(\int_{t \wedge \tau_{d}}^{\tau \wedge \tau_{d}} d A_{u} \mid \mathcal{G}_{t}\right)=1_{\left\{t<\tau_{d}\right\}} e^{\Gamma_{t}} \mathbb{E}_{\mathbb{Q}}\left(\int_{t}^{\tau} e^{-\Gamma_{u}} d A_{u} \mid \mathcal{F}_{t}\right)
$$

Proof. (i) Since $\tau \in \mathcal{F}_{t}^{T}$, one has $\mathcal{F}_{t} \subset \mathcal{F}_{\tau} \subset \mathcal{F}_{T}$, hence by Lemma A.2

$$
\begin{aligned}
\mathbb{E}_{\mathbb{Q}}\left(1_{\left\{\tau<\tau_{d}\right\}} \mid \mathcal{G} \mathcal{G}_{t}\right) & =1_{\left\{t<\tau_{d}\right\}} e^{\Gamma_{t}} \mathbb{E}_{\mathbb{Q}}\left(1_{\left\{\tau<\tau_{d}\right\}} \mid \mathcal{F}_{t}\right) \\
& =1_{\left\{t<\tau_{d}\right\}} e^{\Gamma_{t}} \mathbb{E}_{\mathbb{Q}}\left(X \mathbb{Q}\left(\tau<\tau_{d} \mid \mathcal{F}_{\tau}\right) \mid \mathcal{F}_{t}\right) \\
& =1_{\left\{t<\tau_{d}\right\}} e^{\Gamma_{t}} \mathbb{E}_{\mathbb{Q}}\left(x e^{-\Gamma \tau} \mid \mathcal{F}_{t}\right),
\end{aligned}
$$

where in the last equality one has used (A.6). 
(ii) If suffices to prove the formula for an elementary predictable process of the form $Z_{t}=1_{] u, v]}(t) B_{u}$ for an arbitrary event $B_{u} \in \mathcal{F}_{u}$. For such a process, the formula follows easily from part (i).

(iii) One has that

$$
\int_{t \wedge \tau_{d}}^{\tau \wedge \tau_{d}} d A_{u}=1_{\left\{t<\tau_{d}\right\}} \int_{t \wedge \tau_{d}}^{\tau \wedge \tau_{d}} d A_{u}=1_{\left\{\tau<\tau_{d}\right\}} \int_{t}^{\tau} d A_{u}+1_{\left\{t<\tau_{d} \leq \tau\right\}} \int_{t}^{\tau_{d}} d A_{u}
$$

where $A$ is $\mathbb{F}$-predictable. Using parts (i) and (ii), we obtain

$$
\begin{gathered}
\mathbb{E}_{\mathbb{Q}}\left(1_{\left\{\tau<\tau_{d}\right\}} \int_{t}^{\tau} d A_{u} \mid \mathcal{G}_{t}\right)=1_{\left\{t<\tau_{d}\right\}} \mathbb{E}_{\mathbb{Q}}\left(e^{\Gamma_{t}-\Gamma_{\tau}} \int_{t}^{\tau} d A_{u} \mid \mathcal{F}_{t}\right), \\
\mathbb{E}_{\mathbb{Q}}\left(1_{\left\{t<\tau_{d} \leq \tau\right\}} \int_{t}^{\tau_{d}} d A_{u} \mid \mathcal{G}_{t}\right)=1_{\left\{t<\tau_{d}\right\}} \mathbb{E}_{\mathbb{Q}}\left(\int_{t}^{\tau}\left(\int_{t}^{s} d A_{u}\right) e^{\Gamma_{t}-\Gamma_{s}} d \Gamma_{s} \mid \mathcal{F}_{t}\right),
\end{gathered}
$$

where, by Fubini's theorem,

$$
\int_{t}^{\tau}\left(\int_{t}^{s} d A_{u}\right) \Gamma_{t}-\Gamma_{s} d \Gamma_{S}=\int_{t}^{\tau} \int_{t}^{s} d A_{u} e^{\Gamma_{t}-\Gamma_{s}} d \Gamma_{s}=\int_{t}^{\tau} e^{\Gamma_{t}-\Gamma_{u}} d A_{u}-e^{\Gamma_{t}-\Gamma_{\tau}} \int_{t}^{\tau} d A_{u}
$$

Hence

$$
\mathbb{E}_{\mathbb{Q}}\left(\int_{t \wedge \tau_{d}}^{\tau \wedge \tau_{d}} d A_{u} \mid \mathcal{G}_{t}\right)=1_{\left\{t<\tau_{d}\right\}} \mathbb{E}_{\mathbb{Q}}\left(\int_{t}^{\tau} e^{\Gamma_{t}-\Gamma_{u}} d A_{u} \mid \mathcal{F}_{t}\right)=1_{\left\{t<\tau_{d}\right\}} e^{\Gamma_{t}} \mathbb{E}_{\mathbb{Q}}\left(\int_{t}^{\tau} e^{-\Gamma_{u}} d A_{u} \mid \mathcal{F}_{t}\right),
$$

as was expected.

In the next result, $\left[M_{\cdot \wedge \tau_{d}}, N^{d}\right]$ refers to the square bracket of $M_{\cdot \wedge \tau_{d}}$ and $N^{d}$ with respect to the filtration $\mathbb{G}$, where $N^{d}$ denotes, as usual, the compensated jump-to-default process. This bracket is well defined, since $N^{d}$ is a $\mathbb{G}$-martingale and $M_{\cdot \wedge \tau_{d}}$ is a $\mathbb{G}$-local martingale, by Lemma A.1(iii).

Lemma A.6. For any $\mathbb{F}$-martingale $M$, the process $\left[M_{\cdot \wedge \tau_{d}}, N^{d}\right]$ is a $\mathbb{G}$-local martingale.

Proof. Let us write $H^{d}=(1-H) e^{\Gamma}$. Since $\Gamma$ is continuous and nondecreasing, one has that $d H_{t}^{d}=-H_{t-}^{d} d N_{t}^{d}$ (see [29]). By an application of Lemma A.5(i) with $\tau=T$ and $x=e^{\Gamma_{T}} M_{T}$, we obtain, for every $t \in[0, T]$,

$$
H_{t}^{d} M_{t \wedge \tau_{d}}=1_{\left\{t<\tau_{d}\right\}} e^{\Gamma_{t}} M_{t}=1_{\left\{t<\tau_{d}\right\}} e^{\Gamma_{t}} \mathbb{E}_{\mathbb{Q}}\left(M_{T} \mid \mathcal{F}_{t}\right)=\mathbb{E}_{\mathbb{Q}}\left(1_{\left\{T<\tau_{d}\right\}} e^{\Gamma_{T}} M_{T} \mid \mathcal{G}_{t}\right),
$$


so $M_{t \cdot \wedge \tau_{d}} H_{t}^{d}, t \in[0, T]$, is a $\mathbb{G}$-uniformly integrable martingale, hence $\left[M_{\cdot \wedge \tau_{d}}, H^{d}\right]$ is a $\mathbb{G}$-local martingale (since $M_{\cdot \wedge \tau_{d}}$ and $H^{d}$ are $\mathbb{G}$-local martingales). Now one has that

$$
\left[M_{\cdot \wedge \tau_{d}}, N^{d}\right]_{t}=\Delta M_{\tau_{d}} H_{t}=-e^{-\Gamma_{t}}\left[M_{t \wedge \tau_{d}}, H^{d}\right]_{t^{\prime}}
$$

and thus the conclusion follows.

\section{Acknowledgments}

The research of T. R. Bielecki was supported by NSF Grant 0202851 and Moody's Corporation grant 5-55411. The research of S. Crépey was supported by Ito33 and the Europlace Institute of Finance. The research of M. Jeanblanc was supported by Ito33, FIRN, and Moody's Corporation grant 5-55411 and Fédération Bancaire Française. The research of M. Rutkowski was supported by the ARC Discovery Project DP0881460.

\section{References}

[1] L. Andersen and L. Buffum, "Calibration and implementation of convertible bond models," The Journal of Computational Finance, vol. 7, no. 4, pp. 1-34, 2004.

[2] E. Ayache, P. Forsyth, and K. Vetzal, "Valuation of convertible bonds with credit risk," The Journal of Derivatives, vol. 11, no. 1, pp. 9-29, 2003.

[3] T. R. Bielecki, S. Crépey, M. Jeanblanc, and M. Rutkowski, "Arbitrage pricing of defaultable game options with applications to convertible bonds," Quantitative Finance, vol. 8, no. 8, pp. 795-810, 2008.

[4] T. R. Bielecki, S. Crépey, M. Jeanblanc, and M. Rutkowski, "Convertible bonds in a defaultable diffusion model," Working paper, 2007, http:/ / www.defaultrisk.com.

[5] M. Davis and F. R. Lischka, "Convertible bonds with market risk and credit risk," in Applied Probability, R. Chan, Y.-K. Kwok, D. Yao, and Q. Zhang, Eds., vol. 26 of Studies in Advanced Mathematics, pp. 45-58, American Mathematical Society/International Press, Providence, RI, USA, 2002.

[6] J. Kallsen and C. Kühn, "Convertible bonds: financial derivatives of game type," in Exotic Option Pricing and Advanced Lévy Models, A. Kyprianou, W. Schoutens, and P. Wilmott, Eds., pp. 277-291, John Wiley \& Sons, Chichester, UK, 2005.

[7] Y. Kwok and K. Lau, "Anatomy of option features in convertible bonds," Journal of Futures Markets, vol. 24, no. 6, pp. 513-532, 2004.

[8] Y. Kifer, "Game options," Finance and Stochastics, vol. 4, no. 4, pp. 443-463, 2000.

[9] T. R. Bielecki, S. Crépey, M. Jeanblanc, and M. Rutkowski, "Defaultable options in a Markovian intensity model of credit risk," Mathematical Finance, vol. 18, no. 4, pp. 493-518, 2008.

[10] A. N. Shiryaev and A. S. Cherny, "Vector stochastic integral and the fundamental theorem of asset pricing," Proceedings of the Steklov Mathematical Institute, vol. 237, pp. 12-56, 2002.

[11] M. Chatelain and C. Stricker, "On componentwise and vector stochastic integration," Mathematical Finance, vol. 4, no. 1, pp. 57-65, 1994.

[12] J. Jacod, "Intégrales stochastiques par rapport à une semimartingale vectorielle et changements de filtration," in Séminaire de Probabilités XIV (Paris, 1978/1979), vol. 784 of Lecture Notes in Mathematics, pp. 161-172, Springer, Berlin, Germany, 1980.

[13] S.-W. He, J.-G. Wang, and J.-A. Yan, Semimartingale Theory and Stochastic Calculus, Science Press, Beijing, China; CRC Press, Boca Raton, Fla, USA, 1992.

[14] F. Delbaen and W. Schachermayer, "The fundamental theorem of asset pricing for unbounded stochastic processes," Mathematische Annalen, vol. 312, no. 2, pp. 215-250, 1998.

[15] J. Jacod and A. N. Shiryaev, Limit Theorems for Stochastic Processes, vol. 288 of Grundlehren der Mathematischen Wissenschaften, Springer, Berlin, Germany, 2nd edition, 2003.

[16] P. E. Protter, Stochastic Integration and Differential Equations, vol. 21 of Applications of Mathematics, Springer, Berlin, Germany, 2nd edition, 2004.

[17] E. B. Dynkin, "Game variant of a problem on optimal stopping," Soviet Mathematics Doklady, vol. 10, pp. 270-274, 1969. 
[18] Y. Kifer, "Optimal stopping in games with continuous time," Theory of Probability and Its Applications, vol. 16, pp. 545-550, 1971.

[19] J.-P. Lepeltier and M. A. Maingueneau, “Le jeu de Dynkin en théorie générale sans l'hypothèse de Mokobodski," Stochastics, vol. 13, no. 1-2, pp. 25-44, 1984.

[20] I. Karatzas, "On the pricing of American options," Applied Mathematics and Optimization, vol. 17, no. 1, pp. 37-60, 1988.

[21] N. El Karoui and M. C. Quenez, "Non-linear pricing theory and backward stochastic differential equations," in Financial Mathematics (Bressanone, 1996), W. Runggaldier, Ed., vol. 1656 of Lecture Notes in Mathematics, pp. 191-246, Springer, Berlin, Germany, 1997.

[22] J. Ma and J. Cvitanić, "Reflected forward-backward SDEs and obstacle problems with boundary conditions," Journal of Applied Mathematics and Stochastic Analysis, vol. 14, no. 2, pp. 113-138, 2001.

[23] S. Hamadène, "Mixed zero-sum stochastic differential game and American game options," SIAM Journal on Control and Optimization, vol. 45, no. 2, pp. 496-518, 2006.

[24] M. Schweizer, "Option hedging for semimartingales," Stochastic Processes and Their Applications, vol. 37, no. 2, pp. 339-363, 1991.

[25] T. Jeulin, Semi-Martingales et Grossissement d'une Filtration, vol. 833 of Lecture Notes in Mathematics, Springer, Berlin, Germany, 1980.

[26] C. Dellacherie and P.-A. Meyer, Probabilities and Potential. B: Theory of Martingales, vol. 72 of NorthHolland Mathematics Studies, North-Holland, Amsterdam, The Netherlands, 1982.

[27] D. Coculescu and A. Nikeghbali, "Hazard processes and martingale hazard processes," submitted.

[28] A. Nikeghbali and M. Yor, "A definition and some characteristic properties of pseudo-stopping times," The Annals of Probability, vol. 33, no. 5, pp. 1804-1824, 2005.

[29] T. R. Bielecki and M. Rutkowski, Credit Risk: Modelling, Valuation and Hedging, Springer Finance, Springer, Berlin, Germany, 2002.

[30] J. Cvitanić and I. Karatzas, "Backward stochastic differential equations with reflection and Dynkin games," The Annals of Probability, vol. 24, no. 4, pp. 2024-2056, 1996.

[31] S. Hamadène and M. Hassani, "BSDEs with two reflecting barriers driven by a Brownian and a Poisson noise and related Dynkin game," Electronic Journal of Probability, vol. 11, pp. 121-145, 2006.

[32] S. Crépey, "About the pricing equations in finance," in Paris-Princeton Lectures on Mathematical Finance, Springer, Berlin, Germany, 2009.

[33] S. Crépey and A. Matoussi, "Reflected and doubly reflected BSDEs with jumps: a priori estimates and comparison," The Annals of Applied Probability, vol. 18, no. 5, pp. 2041-2069, 2008.

[34] T. R. Bielecki, S. Crépey, M. Jeanblanc, and M. Rutkowski, "Valuation and hedging of defaultable game options in a hazard process model," Working paper, 2007, http:/ /www.defaultrisk.com.

[35] S. Hamadène, J.-P. Lepeltier, and A. Matoussi, "Double barriers reflected backward SDE's with continuous coefficients," Pitman Research Notes in Mathematics Series, vol. 364, pp. 115-128, 1997.

[36] Y. Dolinsky and Y. Kifer, "Hedging with risk for game options in discrete time," Stochastics, vol. 79, no. 1-2, pp. 169-195, 2007.

[37] C. Dellacherie, B. Maisonneuve, and P.-A. Meyer, Probabilités et Potentiel, Chapitres XVII-XXIV, Hermann, Paris, France, 1992.

[38] A. Nikeghbali, "An essay on the general theory of stochastic processes," Probability Surveys, vol. 3, pp. 345-412, 2006.

[39] C. Dellacherie, Capacités et Processus Stochastiques, Ergebnisse der Mathematik und ihrer Grenzgebiete, Band 6, Springer, Berlin, Germany, 1972. 


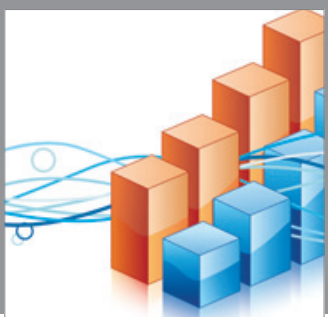

Advances in

Operations Research

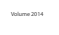

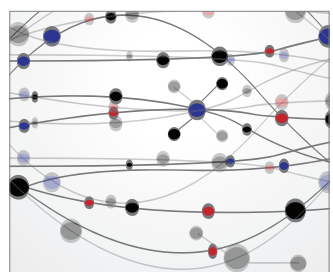

\section{The Scientific} World Journal
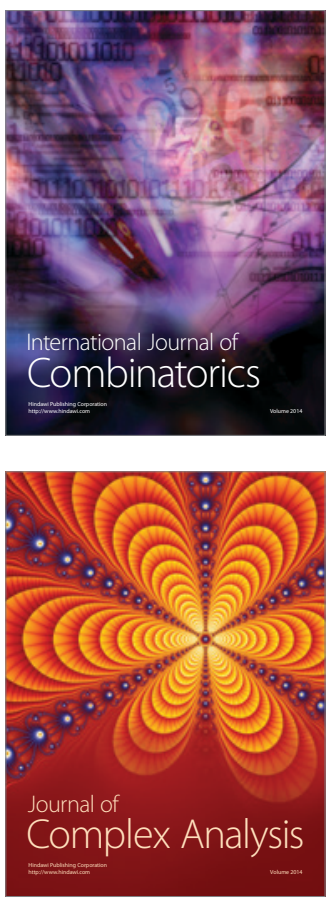

International Journal of

Mathematics and

Mathematical

Sciences
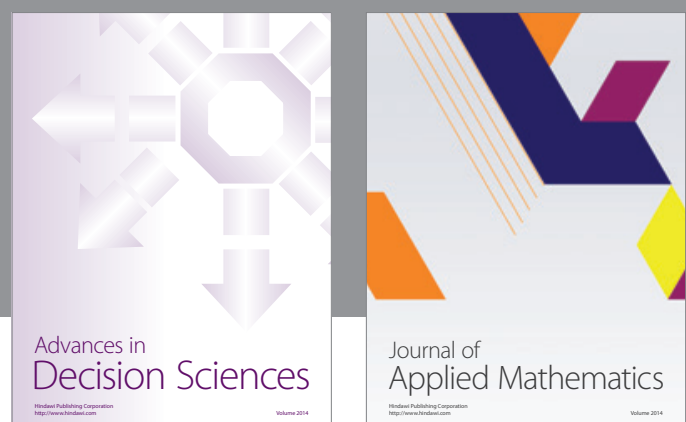

Journal of

Applied Mathematics
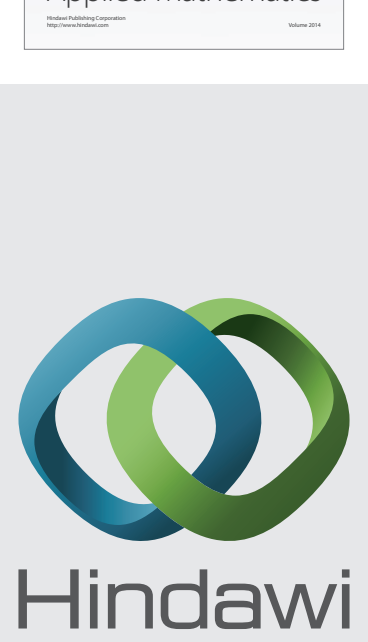

Submit your manuscripts at http://www.hindawi.com
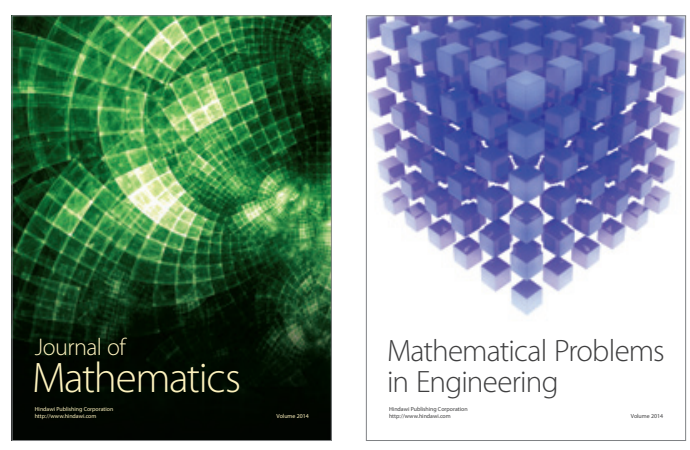

Mathematical Problems in Engineering
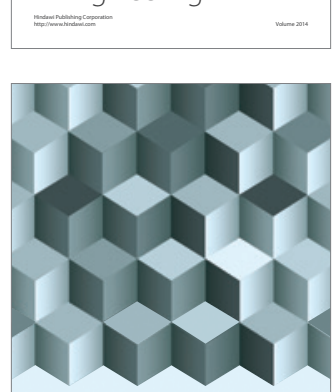

Journal of

Function Spaces
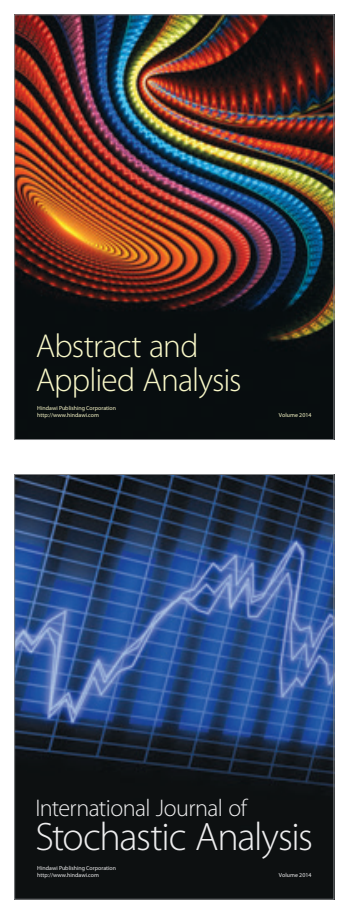

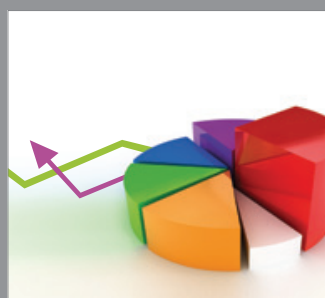

ournal of

Probability and Statistics

Promensencen
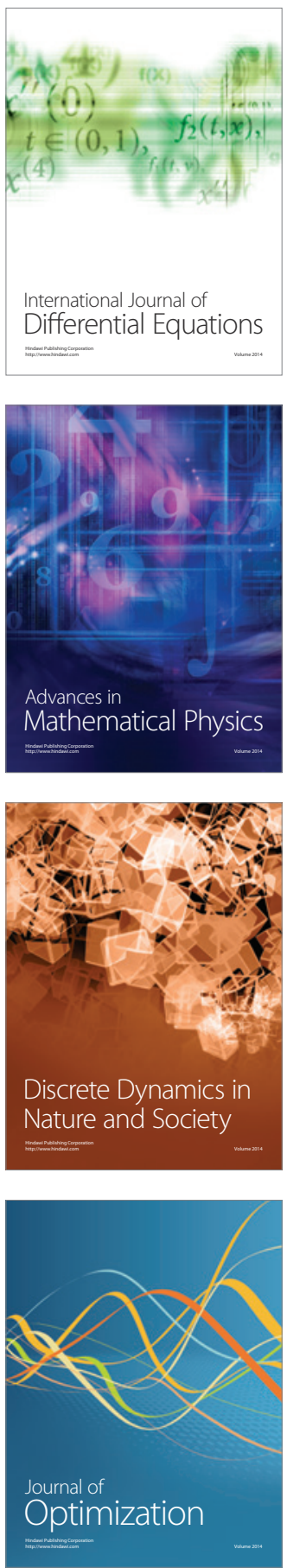